\title{
Band structure effects on the interaction of charged particles with solids
}

\author{
J. M. Pitarke ${ }^{1,2}$ and I. Campillo ${ }^{1}$ \\ ${ }^{1}$ Materia Kondentsatuaren Fisika Saila, Zientzi Fakultatea, Euskal Herriko Unibertsitatea, \\ 644 Posta Kutxatila, 48080 Bilbo, Basque Country, Spain \\ ${ }^{2}$ Donostia International Physics Center (DIPC) and Centro Mixto CSIC-UPV/EHU, \\ Donostia, Basque Country, Spain
}

(June 21, 2021)

\begin{abstract}
A survey is presented of current investigations of the impact of band structure effects on various aspects of the interaction of charged particles with real solids. The role that interband transitions play in the decay mechanism of bulk plasmons is addressed, and results for plasmon linewidths in $\mathrm{Al}$ and $\mathrm{Si}$ are discussed. Ab initio calculations of the electronic energy loss of ions moving in $\mathrm{Al}$ and $\mathrm{Si}$ are also presented, within linear response theory, from a realistic description of the one-electron band structure and a full treatment of the dynamic electronic response of valence electrons. Both random and position-dependent stopping powers of valence electrons are computed.
\end{abstract}

\section{INTRODUCTION}

A quantitative description of the interaction of charged particles with solids is of fundamental importance in a variety of theoretical and applied areas [1]. When a moving charged particle penetrates a solid material, it may lose energy to the medium through various elastic and inelastic collision processes that are based on electron excitation and nuclear recoil motion in the solid. While energy losses due to nuclear recoil may become dominant at very low energies of the projectile [2], in the case of electrons or ions moving with non-relativistic velocities that are comparable to the mean speed of electrons in the solid the most significant energy losses are due to the generation of electronic excitations, such as electron-hole pairs, collective oscillations, i.e., plasmons, and inner-shell excitations and ionizations.

For many years, theoretical investigations of valenceelectron excitations in metals have been carried out on the basis of the so-called jellium model of the solid. Within this model, valence electrons are described by a homogeneous assembly of free electrons immersed in a uniform background of positive charge, the only parameter being the valence-electron density $n_{0}$ or, equivalently, the so-called electron-density parameter $r_{s}$ defined by the relation $1 / n_{0}=(4 / 3) \pi\left(r_{s} a_{0}\right)^{3}, a_{0}$ being the Bohr radius. On the other hand, a widely used approach to treat long-range Coulomb interactions in a many-electron system is the so-called random-phase approximation (RPA) [3], a mean-field theory that has been proved to be successful in describing both electron-hole and plasmon excitations. For instance, the free-electron gas (FEG) predicts, within RPA, a quadratic plasmon dispersion $\omega(q)$ with momentum transfer which is in qualitative agreement with many experimental results 何.

Among the limitations of a free-electron-gas description of the solid there is the absence, within RPA, of any damping mechanism other than the Landau damping where plasmons can decay, for momentum transfers above the critical value $q_{c}$, through the creation of an electron-hole pair. As a result, the FEG predicts, within RPA, an infinitely sharp plasmon line for momentum transfers smaller than $q_{c}$, while measured plasmon lines of real solids show a finite linewidth [4].

Plasmon-decay mechanisms are due, within a freeelectron-gas description of the solid, to the existence of frequency-dependent many-body interactions beyond RPA. These high-order interactions yield non-zero probabilities for the plasmon to decay into two electron-hole pairs or into a plasmon of lower energy plus an electronhole pair, thereby satisfying momentum and energy conservation for arbitrarily small values of $\mathbf{q}$. However, this plasmon damping, which has been shown to be of order $q^{2}$ for small values of the wave vector, cannot explain the observed finite width for $q \rightarrow 0$. Also, calculations by DuBois and Kivelson [5] and by Hasegawa and Watabe [6] show that this contribution to the plasmon linewidth is too small to account alone for the linewidth dispersion in simple metals.

Additional plasmon-decay mechanisms, which yield a finite plasmon linewidth at zero and non-zero momentum transfers, are due to either phonon/impurity assisted electron-hole excitations or to band-structure effects. The former can be accounted by simply introducing a phenomenological relaxation time in the particleconserving RPA-like Mermin dielectric function [7]. The later requires the calculation of the wave-vector and frequency dependent dielectric matrix in the reduced translational symmetry of the real solid. Early calculations of band-structure effects on the plasmon lifetime in simple metals were performed by Sturm and Oliveira [8 10], with the use of a nearly free-electron pseudopotential theory. $A b$ initio calculations of the plasmon lifetime have been carried out only very recently for the case of potassium [11, thereby explaining the experimentally observed anomalous dispersion of the plasmon linewidth in this simple metal [12].

Calculations of the electronic stopping power of solids, i.e., the energy that moving charged particles loose per unit path-length due to electronic excitations in the solid, 
have also been carried out, for many years, on the basis of a jellium model of the target $[13$. The calculation of the stopping power of real solids from the knowledge of the band structure and the corresponding Bloch eigenfunctions is a laborious problem, and early theoretical investigations were based on either semiempirical treatments of the electronic excitations in the solid 14 18 or approximate schemes based on the assumption that electrons are individually bound by harmonic forces [19]. Among the most recent attempts to introduce the full electronic band structure in the evaluation of the electronic stopping power for low projectile velocities there is, for alkaline metals, a one-band calculation [20, as well as a calculation based on a linear combination of atomic orbitals (LCAO) 21]. The low-velocity limit was also investigated, in the case of silicon, on the basis of a static treatment of the density-response of the solid [22], and, more recently, $a b$ initio band structure calculations that are based on a full evaluation of the dynamical densityresponse of the solid have been carried out for aluminum 23,24 and silicon 25].

In this paper we summarize current investigations of the impact of band structure effects on various aspects of the interaction of charged particles with real solids. In Sec. II, we describe our full treatment of the dynamical density-response of valence electrons which is based, in the framework of time-dependent density-functional theory (TDDFT) [26,27, on a realistic description of the one-electron band structure and first-principles pseudopotential theory. In Sec. III, band-structure effects on the energy-loss function are investigated. For wave vectors below the plasmon cutoff $q_{c}$ we focus on both the dispersion of plasmon energy and plasmon linewidths, and for larger wave vectors we investigate the so-called dynamic structure factor. In Sec. IV, the electronic stopping power of valence electrons in aluminum and silicon is considered, which we evaluate within either random or channeling conditions. In Sec. V, our conclusions are presented. In the present work, we restrict our attention to the lowest order in the projectile charge, thereby high-order corrections 28] being ignored. Hence, existing differences between the stopping power for protons and antiprotons are not accounted here, and we focus on the impact of band structure effects on the stopping power of valence electrons in real solids.

\section{LINEAR RESPONSE}

Take a system of interacting electrons exposed to an external potential $V^{e x t}(\mathbf{r}, \omega)$. According to timedependent perturbation theory and keeping only terms of first order in the external perturbation, the electron density induced in the electronic system is found to be

$$
\rho^{i n d}(\mathbf{r}, \omega)=\int \mathrm{d} \mathbf{r}^{\prime} \chi\left(\mathbf{r}, \mathbf{r}^{\prime} ; \omega\right) V^{e x t}\left(\mathbf{r}^{\prime}, \omega\right)
$$

where $\chi\left(\mathbf{r}, \mathbf{r}^{\prime} ; \omega\right)$ represents the so-called linear densityresponse function [3]

$$
\begin{aligned}
\chi\left(\mathbf{r}, \mathbf{r}^{\prime}, \omega\right)=\sum_{n} & \rho_{n 0}^{*}(\mathbf{r}) \rho_{n 0}\left(\mathbf{r}^{\prime}\right)\left[\frac{1}{E_{0}-E_{n}+\hbar(\omega+\mathrm{i} \eta)}\right. \\
& \left.-\frac{1}{E_{0}+E_{n}+\hbar(\omega+\mathrm{i} \eta)}\right]
\end{aligned}
$$

$\eta$ being a positive infinitesimal. $\rho_{n 0}(\mathbf{r})$ represent matrix elements, taken between the unperturbed many-particle ground state $\left|\Psi_{0}\right\rangle$ of energy $E_{0}$ and the unperturbed many-particle excited state $\left|\Psi_{n}\right\rangle$ of energy $E_{n}$, of the particle-density operator

$$
\rho(\mathbf{r})=\sum_{i=1}^{N} \delta\left(\mathbf{r}-\mathbf{r}_{i}\right)
$$

where $\mathbf{r}_{i}$ describe electron coordinates. The inverse dielectric function is connected with the density-response function by the following relation:

$$
\epsilon^{-1}\left(\mathbf{r}, \mathbf{r}^{\prime} ; \omega\right)=\delta\left(\mathbf{r}-\mathbf{r}^{\prime}\right)+\int \mathrm{d} \mathbf{r}^{\prime \prime} v\left(\mathbf{r}-\mathbf{r}^{\prime \prime}\right) \chi\left(\mathbf{r}^{\prime \prime}, \mathbf{r}^{\prime} ; \omega\right),
$$

where $v\left(\mathbf{r}-\mathbf{r}^{\prime}\right)$ is the bare Coulomb interaction.

In the framework of TDDFT, the exact densityresponse function $\chi\left(\mathbf{r}, \mathbf{r}^{\prime} ; \omega\right)$ obeys the integral equation 26

$$
\begin{gathered}
\chi\left(\mathbf{r}, \mathbf{r}^{\prime} ; \omega\right)=\chi^{0}\left(\mathbf{r}, \mathbf{r}^{\prime} ; \omega\right)+\int \mathrm{d} \mathbf{r}_{1} \int \mathrm{d} \mathbf{r}_{2} \chi^{0}\left(\mathbf{r}, \mathbf{r}_{1} ; \omega\right) \\
\times\left[v\left(\mathbf{r}_{1}-\mathbf{r}_{2}\right)+K^{x c}\left(\mathbf{r}_{1}, \mathbf{r}_{2} ; \omega\right)\right] \chi\left(\mathbf{r}_{2}, \mathbf{r}^{\prime} ; \omega\right),
\end{gathered}
$$

where $\chi^{0}\left(\mathbf{r}, \mathbf{r}^{\prime} ; \omega\right)$ represents the density-response function for non-interacting Kohn-Sham electrons [29], and $K^{x c}\left(\mathbf{r}, \mathbf{r}^{\prime} ; \omega\right)$ represents the reduction in the electronelectron interaction due to the existence of dynamical short-range exchange and correlation (XC) effects.

In the case of a homogeneous electron gas, one introduces Fourier transforms and writes

$$
\chi_{\mathbf{q}, \omega}=\chi_{\mathbf{q}, \omega}^{0}+\chi_{\mathbf{q}, \omega}^{0}\left(v_{\mathbf{q}}+K_{\mathbf{q}, \omega}^{x c}\right) \chi_{\mathbf{q}, \omega}
$$

where $v_{\mathbf{q}}$ represents the Fourier transform of the bare Coulomb interaction, $\chi_{\mathbf{q}, \omega}^{0}$ is the well-known function of Lindhard 30], and

$$
K_{\mathbf{q}, \omega}^{x c}=-v_{\mathbf{q}} G_{\mathbf{q}, \omega}
$$

$G_{\mathbf{q}, \omega}$ being the so-called local-field factor [31 33.

For a periodic crystal, we introduce the following Fourier expansion of the density-response function:

$$
\chi\left(\mathbf{r}, \mathbf{r}^{\prime} ; \omega\right)=\frac{1}{V} \sum_{\mathbf{q}}^{B Z} \sum_{\mathbf{G}, \mathbf{G}^{\prime}} \mathrm{e}^{\mathrm{i}(\mathbf{q}+\mathbf{G}) \cdot \mathbf{r}} \mathrm{e}^{-\mathrm{i}\left(\mathbf{q}+\mathbf{G}^{\prime}\right) \cdot \mathbf{r}^{\prime}} \chi_{\mathbf{G}, \mathbf{G}^{\prime}}(\mathbf{q}, \omega),
$$


where $V$ represents the normalization volume, the first sum runs over $\mathbf{q}$ vectors within the first Brillouin zone (BZ), and $\mathbf{G}$ and $\mathbf{G}^{\prime}$ are reciprocal lattice vectors. Introduction of Eq. (8) into Eqs. (4) and (5) yields matrix equations for the Fourier coefficients $\chi_{\mathbf{G}, \mathbf{G}^{\prime}}(\mathbf{q}, \omega)$ and $\epsilon_{\mathbf{G}, \mathbf{G}^{\prime}}^{-1}(\mathbf{q}, \omega)$. In the case of non-interacting Kohn-Sham electrons, one finds:

$$
\begin{aligned}
& \chi_{\mathbf{G}, \mathbf{G}^{\prime}}^{0}(\mathbf{q}, \omega)=\frac{1}{V} \sum_{\mathbf{k}}^{B Z} \sum_{n, n^{\prime}} \frac{f_{\mathbf{k}, n}-f_{\mathbf{k}+\mathbf{q}, n^{\prime}}}{E_{\mathbf{k}, n}-E_{\mathbf{k}+\mathbf{q}, n^{\prime}}+\hbar(\omega+\mathrm{i} \eta)} \\
& \times\left\langle\phi_{\mathbf{k}, n}\left|e^{-\mathrm{i}(\mathbf{q}+\mathbf{G}) \cdot \mathbf{r}}\right| \phi_{\mathbf{k}+\mathbf{q}, n^{\prime}}\right\rangle\left\langle\phi_{\mathbf{k}+\mathbf{q}, n^{\prime}}\left|e^{\mathrm{i}\left(\mathbf{q}+\mathbf{G}^{\prime}\right) \cdot \mathbf{r}}\right| \phi_{\mathbf{k}, n}\right\rangle .
\end{aligned}
$$

The second sum runs over the band structure for each wave vector $\mathbf{k}$ in the first Brillouin zone, $f_{\mathbf{k}, n}$ represents the Fermi-Dirac distribution function, and $\phi_{\mathbf{k}, n}(\mathbf{r})$ and $E_{\mathbf{k}, n}$ are Bloch eigenfunctions and eigenvalues of the Kohn-Sham Hamiltonian of density-functional theory (DFT) 29], which we evaluate within the local-density approximation (LDA) with use of the parametrization of Perdew and Zunger 34. All results presented in this article have been found to be well converged with the use in Eq. (9) of $60(\mathrm{Al})$ and $200(\mathrm{Si})$ bands. The sum over the Brillouin zone has been performed with the use of $10 \times 10 \times 10$ and $8 \times 8 \times 8$ Monkhorst-Pack meshes 35 for $\mathrm{Al}$ and $\mathrm{Si}$, respectively.

For the evaluation of the one-electron $\phi_{\mathbf{k}, n}(\mathbf{r})$ eigenfunctions, we first expand them in a plane-wave basis,

$$
\phi_{\mathbf{k}, n}(\mathbf{r})=\frac{1}{\sqrt{V}} \sum_{\mathbf{G}} u_{\mathbf{k}, n}(\mathbf{G}) \mathrm{e}^{\mathrm{i}(\mathbf{k}+\mathbf{G}) \cdot \mathbf{r}}
$$

with a kinetic-energy cutoff that varies from $12 \mathrm{Ry}$ in the case of $\mathrm{Al}(\sim 100 \mathrm{G}$-vectors $)$ to $16 \mathrm{Ry}$ in the case of Si ( 300 G-vectors). Then, we evaluate the coefficients $u_{\mathbf{k}, n}$ by solving the Kohn-Sham equation of DFT with a full description of the electron-ion interaction that is based on the use of an ab initio non-local, normconserving ionic pseudopotential 36]. Finally, we evaluate from Eq. (9) the Fourier coefficients of the noninteracting density-response function, and solve a matrix equation for the Fourier coefficients of the interacting density-response function, which we obtain within either RPA or TDLDA.

In the RPA, the XC kernel $K^{x c}\left(\mathbf{r}, \mathbf{r}^{\prime} ; \omega\right)$ entering Eq. (5) is set equal to zero. Within TDLDA, which represents an adiabatic extension to finite frequencies of the LDA, one writes

$$
K_{x c}^{L D A}\left(\mathbf{r}, \mathbf{r}^{\prime} ; \omega\right)=\delta\left(\mathbf{r}-\mathbf{r}^{\prime}\right)\left[\frac{\mathrm{d} V_{x c}(n)}{\mathrm{d} n}\right]_{n=n_{0}(\mathbf{r})},
$$

where $V_{x c}(n)$ represents the LDA XC potential entering the Kohn-Sham equation of DFT, and $n_{0}(\mathbf{r})$ is the actual density of the electron system. In the case of a homogeneous electron gas of density $n_{0}$, the Fourier transform of the XC kernel of Eq. (11) can be written in the form of Eq. (7) with

$$
G_{\mathbf{q}, \omega}^{L D A}=A\left(\frac{q}{q_{F}}\right)^{2}
$$

where

$$
A=\frac{1}{4}-\frac{4 \pi a_{0}^{2}}{e^{2} q_{F}^{2}}\left[\frac{\mathrm{d} V_{c}(n)}{\mathrm{d} n}\right]_{n=n_{0}}
$$

$V_{c}(n)$ being the correlation contribution to $V_{x c}$, and $q_{F}$, the Fermi momentum.

\section{ENERGY-LOSS FUNCTION}

\section{A. Theory}

The Hamiltonian describing the interaction between a particle of charge $Z_{1} e$ at point $\mathbf{r}$ and the many-electron system is given by

$$
H_{I}=-Z_{1} e^{2} \int d \mathbf{r}^{\prime} \frac{\rho\left(\mathbf{r}^{\prime}\right)}{\left|\mathbf{r}-\mathbf{r}^{\prime}\right|}
$$

where $\rho(\mathbf{r})$ represents the particle-density operator of Eq. (3).

Within first-order perturbation theory, the probability per unit time for the probe particle to scatter from an initial state $\mid i>$ of energy $\varepsilon_{i}$ to a final state $\mid f>$ of energy $\varepsilon_{f}$, by carrying the Fermi gas from the manyparticle ground state $\mid \Psi_{0}>$ of energy $E_{0}$ to some excited many-particle state $\left|\Psi_{n}\right\rangle$ of energy $E_{n}$, is given by the following expression:

$P_{i \rightarrow f}=\frac{2 \pi}{\hbar} \sum_{n}\left|<\Psi_{n} f\right| H_{I}\left|\Psi_{0} i>\right|^{2} \delta\left(\varepsilon_{i}-\varepsilon_{f}+E_{0}-E_{n}\right)$.

If one chooses the probe particle to be described by plane-wave states

$$
\phi_{i}(\mathbf{r})=\frac{1}{\sqrt{V}} \mathrm{e}^{\mathrm{i} \mathbf{q}_{i} \cdot \mathbf{r}}
$$

and

$$
\phi_{f}(\mathbf{r})=\frac{1}{\sqrt{V}} \mathrm{e}^{\mathrm{i} \mathbf{q}_{f} \cdot \mathbf{r}},
$$

one finds the probability per unit time for the probe particle to transfer momentum $\hbar \mathbf{q}=\hbar\left(\mathbf{q}_{i}-\mathbf{q}_{f}\right)$ to the Fermi gas to be given by the following expression:

$$
P_{\mathbf{q}}=\frac{2 \pi}{(\hbar V)^{2}} Z_{1}^{2} v_{\mathbf{q}}^{2} S(\mathbf{q}, \omega),
$$

where $\hbar \omega$ represents the energy transfer,

$$
\hbar \omega=\frac{\hbar^{2}}{2 m_{e}}\left(k_{i}^{2}-k_{f}^{2}\right),
$$


and $S(\mathbf{q}, \omega)$ is the so-called dynamic structure factor of the many-electron system:

$$
S(\mathbf{q}, \omega)=\sum_{n}\left|\left(\rho_{\mathbf{q}}^{\dagger}\right)_{n 0}\right|^{2} \delta\left(\omega-\omega_{n 0}\right) .
$$

Here, $\rho_{\mathbf{q}}$ represents the Fourier transform of the particle density, $\left(\rho_{\mathbf{q}}^{\dagger}\right)_{n 0}$ are matrix elements taken between the many-particle eigenstates $\left|\Psi_{0}\right\rangle$ and $\left|\Psi_{n}\right\rangle$, and $\hbar \omega_{n 0}=$ $E_{n}-E_{0}$.

The double differential cross section $\mathrm{d}^{2} \sigma / \mathrm{d} \Omega \mathrm{d} \omega$ for inelastic scattering of charged particles is simply the number of particles being scattered per unit time, unit solid angle, and unit frequency into the solid angle $\Omega$ with energy transfer $\hbar \omega$, divided by the initial particle flux. From Eq. (18), one easily finds

$$
\frac{\mathrm{d}^{2} \sigma}{\mathrm{d} \Omega \mathrm{d} \omega}=\frac{Z_{1}^{2}}{4 \pi^{2} a_{0}^{2} e^{4}} \frac{q_{f}}{q_{i}} v_{\mathbf{q}}^{2} S(\mathbf{q}, \omega) .
$$

While high-energy transmission electron beams have been used to probe $S(\mathbf{q}, \omega)$ for $q<q_{F}$, thereby providing experimental evidence of collective excitations [4], higher values of $\mathbf{q}$ have been studied with use of inelastic X-ray scattering [37]. Within the lowest-order Born approximation, the inelastic $\mathrm{X}$-ray scattering cross section is given by

$$
\frac{\mathrm{d}^{2} \sigma}{\mathrm{d} \Omega \mathrm{d} \omega}=\left(\mathbf{e}_{0} \cdot \mathbf{e}_{1}\right)^{2} \pi r_{0}^{2} \frac{\omega_{1}}{\omega_{0}} S(\mathbf{q}, \omega)
$$

where $\left(\mathbf{e}_{0}, \omega_{0}\right)$ and $\left(\mathbf{e}_{0}, \omega_{0}\right)$ are the polarization and frequency of the incident and scattered photon, respectively, and $r_{0}$ is the classical electron radius.

The dynamic structure factor, which determines the fluctuation of the particle density, is connected to the imaginary part of the density-response function of Eq. (2) through the fluctuation-dissipation theorem. At zero temperature, one writes 38:

$$
S(\mathbf{q}, \omega)=-\frac{\hbar}{\pi} \int \mathrm{d} \mathbf{r} \int \mathrm{d} \mathbf{r}^{\prime} \mathrm{e}^{-\mathrm{i} \mathbf{q} \cdot\left(\mathbf{r}-\mathbf{r}^{\prime}\right)} \operatorname{Im} \chi\left(\mathbf{r}, \mathbf{r}^{\prime} ; \omega\right)
$$

Hence, in the case of a uniform electron gas one easily finds the absorption probability $P_{\mathbf{q}}$ to be given by the following expression:

$$
P_{\mathbf{q}}=\frac{2}{\hbar V} Z_{1}^{2} v_{\mathbf{q}} \operatorname{Im}\left[-\epsilon^{-1}(\mathbf{q}, \omega)\right],
$$

where $\operatorname{Im}\left[-\epsilon^{-1}(\mathbf{q}, \omega)\right]$ is the so-called energy-loss function of a homogeneous electron gas. For periodic crystals, introduction of Eq. (8) into Eq. (23) yields:

$$
P_{\mathbf{q}+\mathbf{G}}=\frac{2}{\hbar V} Z_{1}^{2} v_{\mathbf{q}+\mathbf{G}} \operatorname{Im}\left[-\epsilon_{\mathbf{G}, \mathbf{G}}^{-1}(\mathbf{q}, \omega)\right]
$$

where $\mathbf{q}$ represents a wave vector in the first BZ, $\hbar(\mathbf{q}+\mathbf{G})$ is the momentum transfer, and $\epsilon_{\mathbf{G}, \mathbf{G}^{\prime}}^{-1}(\mathbf{q}, \omega)$ is the inverse dielectric matrix:

$$
\epsilon_{\mathbf{G}, \mathbf{G}^{\prime}}^{-1}(\mathbf{q}, \omega)=\delta_{\mathbf{G}, \mathbf{G}^{\prime}}+v_{\mathbf{q}+\mathbf{G}} \chi_{\mathbf{G}, \mathbf{G}^{\prime}}(\mathbf{q}, \omega)
$$

If recoil of the probe particle can be neglected, as in the case of heavy projectiles or fast electrons with small values of the momentum transfer, the energy transfer is simply $\hbar \omega=\hbar \mathbf{q} \cdot \mathbf{v}$.

We note that as long as the probe particle can be described by plane waves, the absorption probability in periodic crystals is proportional to the imaginary part of one diagonal element of the energy-loss matrix $\operatorname{Im}\left[-\epsilon_{\mathbf{G}, \mathbf{G}^{\prime}}^{-1}(\mathbf{q}, \omega)\right]$. Hence, crystalline local-field effects 39,40 , i.e., couplings between $\mathbf{q}+\mathbf{G}$ and $\mathbf{q}+\mathbf{G}^{\prime}$ wave vectors with $\mathbf{G} \neq \mathbf{G}^{\prime}$ only enter through the dependence of the diagonal elements of the inverse dielectric matrix on the off-diagonal elements of the direct matrix.

When the probe particle moves with constant velocity $\mathbf{v}$ on a definite trajectory at a given impact vector $\mathbf{b}$, initial and final states can be described in terms of plane waves in the direction of motion and a $\delta$ function in the transverse direction [24]. As a result, the probability per unit time for the probe particle to transfer momentum $\hbar \mathbf{q}$ to the Fermi gas is now given by the following expression:

$$
P_{\mathbf{q}}=\frac{2 \pi}{(\hbar V)^{2}} Z_{1}^{2} \sum_{\mathbf{q}^{\prime}} v_{\mathbf{q}} v_{\mathbf{q}^{\prime}} \mathrm{e}^{\mathrm{i} \mathbf{b} \cdot\left(\mathbf{q}+\mathbf{q}^{\prime}\right)} S\left(\mathbf{q}, \mathbf{q}^{\prime} ; \omega\right) \delta_{q_{z}-q_{z}^{\prime}},
$$

where $\omega=\mathbf{q} \cdot \mathbf{v}, \delta_{q_{z}-q_{z}^{\prime}}$ represents the Kroenecker $\delta$ symbol, $q_{z}$ and $q_{z}^{\prime}$ are components of $\mathbf{q}$ and $\mathbf{q}^{\prime}$ in the direction of motion, and

$$
S\left(\mathbf{q}, \mathbf{q}^{\prime} ; \omega\right)=-\frac{\hbar}{\pi} \int \mathrm{d} \mathbf{r} \int \mathrm{d} \mathbf{r}^{\prime} \mathrm{e}^{-\mathrm{i}\left(\mathbf{q} \cdot \mathbf{r}+\mathbf{q}^{\prime} \cdot \mathbf{r}^{\prime}\right)} \operatorname{Im} \chi\left(\mathbf{r}, \mathbf{r}^{\prime} ; \omega\right) .
$$

In the case of a homogeneous electron gas the probability $P_{\mathbf{q}}$ of Eq. (27) is easily found to be independent of the impact vector and to coincide with that of Eq. (24). However, for periodic crystals introduction of Eq. (8) into Eq. (28) yields the position-dependent absorption probability:

$$
P_{\mathbf{q}+\mathbf{G}}=\frac{2}{\hbar V} Z_{1}^{2} \sum_{\mathbf{K}}{ }^{\prime} \mathrm{e}^{\mathrm{i} \mathbf{K} \cdot \mathbf{b}} v_{\mathbf{q}+\mathbf{G}+\mathbf{K}} \operatorname{Im}\left[-\epsilon_{\mathbf{G}, \mathbf{G}+\mathbf{K}}^{-1}(\mathbf{q}, \omega)\right],
$$

where the prime in the summation indicates that it is restricted to those reciprocal-lattice vectors that are perpendicular to the velocity of the projectile, i.e., $\mathbf{K} \cdot \mathbf{v}=$ 0 . The most important contribution to the positiondependent probability of Eq. (29) is provided by the term $\mathbf{K}=0$, the magnitude of higher-order terms depending on the direction of the velocity. For those directions for which the condition $\mathbf{K} \cdot \mathbf{v}=0$ is never satisfied we have the absorption probability of Eq. (25), and for a few highly symmetric or channeling directions nonnegligible corrections to the random result are expected. 
We also note that the average over impact parameters of the position-dependent probability of Eq. (29) along any given channel coincides with the absorption probability of Eq. 25.

\section{B. Results and discussion}

First of all, we examine the dispersion of the plasmon energy and linewidth by calculating the electron energyloss function $\operatorname{Im}\left[-\epsilon_{\mathbf{G}, \mathbf{G}}^{-1}(\mathbf{q}, \omega)\right]$ for wave vectors that are below the plasmon cutoff $q_{c}$. For these wave vectors the FEG predicts, within either RPA or TDLDA, sharp plasmon lines, which would be broadened through the introduction of a finite value of $\eta$ in Eq. (9). In order to avoid numerical broadening, we have computed the energy-loss function of the real solid for imaginary frequencies and have obtained it on the real axis with $\eta \rightarrow 0^{+}$by using Padé approximants [11,42].

Our computed RPA plasmon peaks in $\mathrm{Al}$ are displayed in Fig. 1, for increasing magnitude of the wave vector in the (100) direction. Vertical lines are $\delta$ functions corresponding to plasmon excitation in a FEG. As a result of the actual band structure of the solid, the energyloss function exhibits a sharp but finite plasmon peak at low wave vectors with a full width at half maximum (FWHM) that increases rapidly, for increasing $\mathbf{q}$, while the peak height decreases according to the $f$-sum rule. For periodic crystals, one writes:

$$
\int_{0}^{\infty} \mathrm{d} \omega \omega \operatorname{Im}\left[-\epsilon_{\mathbf{G}, \mathbf{G}^{\prime}}^{-1}(\mathbf{q}, \omega)\right]=\frac{2 \pi^{2} e^{2}}{m_{e}} n_{\mathbf{G}-\mathbf{G}^{\prime}}
$$

where $n_{\mathbf{G}}$ represents the Fourier components of the density, and $n_{\mathbf{G}=0}$ equals the average electron density of the crystal.

RPA and TDLDA plasmon peak positions in Al, as calculated along the (100) direction, are plotted in Fig. 2 by solid and dotted lines, respectively, as a function of the magnitude of the wave vector. The calculated plasmon dispersions agree with those of previous calculations 43,44]. For comparison, RPA and TDLDA plasmon dispersions corresponding to a FEG with $r_{s}=2.07$ are represented by dashed and dashed-dotted lines, respectively, showing that band-structure corrections result in a nearly-q-independent downward shift of plasmon energy. If short-range $\mathrm{XC}$ effects, which reduce the plasma frequency for large values of the wave vector, are included within the TDLDA, then one finds a result for the plasmon dispersion that is in excellent agreement with experiment 45.

The plasmon lifetime is defined as the inverse of the FWHM of the energy-loss peak, $\Delta E_{1 / 2}$. RPA and TDLDA calculations of $\Delta E_{1 / 2}$ along the (100) direction in $\mathrm{Al}$ are exhibited in Fig. 3, as a function of the magnitude of the wave vector. In the limit as $q \rightarrow 0$ we find $\Delta E_{1 / 2}(0) \sim 0.2 \mathrm{eV}$, below the early calculation carried out by Sturm with use of the nearly free electron approximation [8]. As for plasmon decay from the creation of two electron-hole pairs, which is not included in our band-structure calculation, one finds to lowest-order in a small- $q$ expansion 10:

$$
\Delta E_{1 / 2}^{\text {pair-pair }}(q)=b\left(q / q_{F}\right)^{2} \hbar \omega_{p},
$$

where $b \sim 0.03$ for $r_{s} \sim 2$. Adding band-structure effects, as derived from our calculated RPA plasmon peaks, and many-body corrections, as obtained from Eq. (31), we have found the results plotted in Fig. 3 by open triangles. Solid circles correspond to the result of not including plasmon decay from many-body effects, thereby showing that these effects give only a minor contribution to the plasmon linewidth. As phonons are expected to give a relative contribution to $\Delta E_{1 / 2}(q)$ that changes little with the magnitude of the wave vector [10], the reduced plasmon linewidth $\Delta E_{1 / 2}(q) / \Delta E_{1 / 2}(0)$ is plotted in Fig. 4 by open circles (RPA) and triangles (TDLDA), showing a reasonable agreement with the experimental results of Gibbons et al [46].

In Fig. 5 we show full-band-structure calculations of RPA and TDLDA energy-loss functions in Si, as obtained along the (111) direction. As in Fig. 1, vertical lines represent $\delta$ functions corresponding to plasmon excitations in a FEG, now with $r_{s}=2.01$. Besides the finite width of the energy-loss function of real $\mathrm{Si}$, which is found to be about 5 times wider than in $\mathrm{Al}$, our calculated plasmon energy shows a characteristic $q$ dependence which is considerably different from that of a FEG.

The energy positions of RPA and TDLDA plasmon peaks of Si are plotted in Fig. 6 along the (111) direction, as a function of the magnitude of the wave vector, together with the FEG prediction and the experimental result 47]. In the long-wavelength limit $(q \rightarrow 0)$, the plasmon energy is expected to be only slightly larger than in the case of a FEG, in agreement with the Penn model dielectric function 48 for semiconductors which predicts a plasmon frequency $\omega=\left(\omega_{g}^{2}+\omega_{p}^{2}\right)^{1 / 2}, \hbar \omega_{g}$ being the energy of the effective band gap and $\omega_{p}$ representing the plasma frequency of the corresponding FEG. We also note that while in the case of $\mathrm{Al}$ band-structure effects result in a nearly-q-independent downward shift of the FEG curve, interband transitions in Si flatten the plasmon curve with a considerable reduction of the plasma frequency at large values of the wave vector. Though our calculated plasmon dispersion in $\mathrm{Si}$ is at low wave vectors in good agreement with the experiment, at large $|\mathbf{q}|$ our predictions start to deviate from the experimental value, a result also found in previous calculations 49,50.

In Fig. 7 we show our calculation of the plasmon linewidth dispersion of $\mathrm{Si}$, as obtained along the (111) direction with no inclusion of crystalline local-field effects. In the limit as $q \rightarrow 0$ we find $\Delta E_{1 / 2}(0) \sim 1 \mathrm{eV}$, well below the prediction of Louie et al 51] who performed a calculation of the energy-loss function in $\mathrm{Si}$ at $\mathbf{q}=0$ with full inclusion of local-field effects. This shows 
the key role that coupling between $\mathbf{q}+\mathbf{G}$ and $\mathbf{q}+\mathbf{G}^{\prime}$ wave vectors with $\mathbf{G} \neq \mathbf{G}^{\prime}$ plays in the plasmon-decay mechanism in Si [25], which is a consequence of the presence of directional covalent bondings in this material. Considerable broadening of plasmon peaks in Si by the effect of local-field effects has been reported recently by Lee and Chang [42].

Next, we calculate the energy-loss function in $\mathrm{Al}$ and Si for large wave vectors of $|\mathbf{q}+\mathbf{G}|=1.65 q_{F}$ and $|\mathbf{q}+\mathbf{G}|=1.95 q_{F}$, respectively, along the directions $(1,5 / 6,5 / 6)$ and $(1,1,1)$. The results we have obtained within RPA and TDLDA are plotted in Figs. 8 and 9, showing in the case of $\mathrm{Al}$ the experimentally determined double-peak structure [37] which was recently explained [52 as originated from band-structure effects.

We note that splitting of the band structure of $\mathrm{Al}$ over the Fermi level opens new channels for the creation of electron-hole pairs, which results in an energy-loss function that is, at low frequencies, higher than in the case of a FEG (see Fig. 8). In the case of $\mathrm{Si}$, the presence of the band gap reduces the energy-loss function (see Fig. 9). As a consequence, both the absorption probability and the electronic stopping power of $\mathrm{Si}$ will be, at low energies, smaller than those of $\mathrm{Al}$, though they both have about the same valence electron density.

\section{ELECTRONIC STOPPING POWER}

The electronic stopping power of a moving particle of charge $Z_{1} e$ is simply the energy that it looses per unit path-length due to electronic excitations in the solid. Hence, one writes

$$
-\frac{\mathrm{d} E}{\mathrm{~d} x}=\frac{1}{v} \sum_{\mathbf{q}}\left[\hbar \omega P_{\mathbf{q}}\right],
$$

where $P_{\mathbf{q}}$ represents the probability per unit time for the projectile to transfer momentum $\hbar \mathbf{q}$ to the electron gas, the sum is extended over all momentum transfers, $\mathbf{v}$ is the projectile velocity, and $\hbar \omega$ is the energy transfer. As long as recoil can be neglected, $\hbar \omega=\hbar \mathbf{q} \cdot \mathbf{v}$.

In the case of a homogeneous FEG the probability $P_{\mathbf{q}}$ is given by Eq. (24), and introduction of this expression into Eq. (32) yields the well-known formula for the stopping power of a FEG [1]. For periodic crystals, the energy loss depends on whether the projectile is moving at a definite impact parameter or not. For random trajectories, introduction of Eq. (25) into Eq. (32) results in the so-called random stopping power:

$$
\begin{aligned}
{\left[-\frac{\mathrm{d} E}{\mathrm{~d} x}\right]_{\text {random }}=} & \frac{Z_{1}^{2}}{4 \pi^{3} v} \int_{\mathrm{BZ}} \mathrm{d} \mathbf{q} \sum_{\mathbf{G}} \omega v_{\mathbf{q}+\mathbf{G}} \\
& \times \operatorname{Im}\left[-\epsilon_{\mathbf{G}, \mathbf{G}}^{-1}(\mathbf{q}, \omega)\right],
\end{aligned}
$$

where the integral runs over $\mathbf{q}$ vectors within the first Brillouin zone, and $\omega=(\mathbf{q}+\mathbf{G}) \cdot \mathbf{v}$.
In the case of charged particles moving with constant velocity $\mathbf{v}$ on a definite trajectory at a given impact parameter $\mathbf{b}$, the probability per unit time for the projectile to transfer momentum $\hbar(\mathbf{q}+\mathbf{G})$ to the electron gas is given by Eq. (29), and introduction of this expression into Eq. (32) yields the following result for the positiondependent stopping power:

$$
\begin{aligned}
{\left[-\frac{\mathrm{d} E}{\mathrm{~d} x}\right]_{\mathbf{b}}=} & \frac{Z_{1}^{2}}{4 \pi^{3} v} \int_{\mathrm{BZ}} \mathrm{d} \mathbf{q} \sum_{\mathbf{G}} \sum_{\mathbf{K}}{ }^{\prime} \omega \mathrm{e}^{\mathrm{i} \mathbf{K} \cdot \mathbf{b}} \\
& \times v_{\mathbf{q}+\mathbf{G}+\mathbf{K}} \operatorname{Im}\left[-\epsilon_{\mathbf{G}, \mathbf{G}+\mathbf{K}}^{-1}(\mathbf{q}, \omega)\right],
\end{aligned}
$$

where the sum $\sum_{\mathbf{K}}^{\prime}$ is restricted, as in Eq. (29), to those reciprocal-lattice vectors that are perpendicular to the projectile velocity, and $\omega=(\mathbf{q}+\mathbf{G}) \cdot \mathbf{v}$, as in Eq. (33).

The main ingredient in the calculation of both random and position-dependent stopping powers is the inverse dielectric matrix $\epsilon_{\mathbf{G}, \mathbf{G}^{\prime}}^{-1}(\mathbf{q}, \omega)$, which has been discussed in the previous section. As the maximum energy that the moving particle may transfer to the target is $(\hbar \omega)_{\max }=q v$, the number of bands that are required in the evaluation of the polarizability $\chi_{\mathbf{G}, \mathbf{G}^{\prime}}^{0}(\mathbf{q}, \omega)$ of Eq. (9) depends on the projectile velocity. Well-converged results have been found for all projectile velocities under study, with the use in Eq. (9) of 60 bands for $\mathrm{Al}$ and 200 bands for Si. The sums over reciprocal-lattice vectors in Eqs. (33) and (34) have been extended over 15 values of reciprocal-lattice $\mathbf{G}$ vectors, the magnitude of the maximum momentum transfer being $2.9 q_{F}$ and $2.1 q_{F}$ for $\mathrm{Al}$ and $\mathrm{Si}$, respectively. All calculations have been performed with full inclusion of crystalline local-field effects, i.e., by inversion of the full dielectric matrix $\epsilon_{\mathbf{G}, \mathbf{G}^{\prime}}(\mathbf{q}, \omega)$, and contributions from these so-called local-field effects to the random stopping power of $\mathrm{Al}$ and $\mathrm{Si}$ have been found to be within $0.5 \%$ and $1 \%$, respectively.

\section{A. Random stopping power}

In Fig. 10 we show, as a function of the projectile velocity, our full RPA and TDLDA results for the random stopping power of valence electrons in real $\mathrm{Al}$ for protons $\left(Z_{1}=1\right)$, together with the corresponding result for the stopping power of a FEG with an electron-density parameter equal to that of $\mathrm{Al}\left(r_{s}=2.07\right)$ [53]. These results have been found to be insensitive to the choice of the projectile-velocity direction.

As the energy-loss function of real $\mathrm{Al}$ is, at low frequencies, slightly enhanced with respect to the corresponding FEG calculation (see Fig. 8), the stopping power of the real target is, for projectile velocities smaller than the Fermi velocity and within both RPA and TDLDA, higher than that of a FEG by about $7 \%$.

At velocities over the plasmon-threshold velocity for which plasmon excitation becomes possible, contributions to the stopping power come from both plasmon 
and electron-hole excitations. These contributions have been calculated separately 24, showing that contributions from losses to plasmon excitation are independent of the detailed band structure of the crystal. As for the contribution from the excitation of electron-hole pairs, band-structure effects in $\mathrm{Al}$ are found to lower the stopping power of electrons in a FEG by about $10 \%$ at and just above the plasmon-threshold velocity.

At high velocities, well above the stopping maximum, the sum over the frequency $\omega$ in Eq. (33) can be replaced by an integration from 0 to $\infty$, and the sum rule of Eq. (30) results in a stopping power which depends on the average electron density $n_{0}$ and not on the details of the band structure of the target material:

$$
\left[-\frac{\mathrm{d} E}{\mathrm{~d} x}\right]_{\text {random }} \sim \frac{4 \pi Z_{1}^{2} e^{4}}{m_{e} v^{2}} n_{0} \ln \frac{2 m_{e} v^{2}}{\hbar \omega_{p}} .
$$

While at low velocities the contribution to the total energy loss due to excitation of inner-shell electrons is negligible small, at velocities larger than the Fermi velocity it is necessary to allow for this contribution. The cross sections for the ionization of inner shells in $\mathrm{Al}$ were obtained by Ashley et al [54 in the first Born approximation utilizing atomic generalized oscillator strength functions. By adding the contribution from core electrons to that of valence electrons (this contribution does not depend, at these velocities, on the details of the band structure) these authors found a nice agreement with experiment. Good agreement with experiment was also shown in Ref. [2] by adding the energy loss from core electrons in $\mathrm{Si}$, as taken from Walske's calculations [55], to that from valence electrons.

Our full calculation of RPA and TDLDA stopping powers of valence electrons in $\mathrm{Si}$ is plotted in Fig. 11, as a function of the projectile velocity, with $Z_{1}=1$, together with the corresponding result for the stopping power of a FEG with $r_{s}=2.01$ [53. As in the case of $\mathrm{Al}$, these results have been found to be insensitive to the choice of the direction of the projectile velocity.

The band gap in Si clearly makes the stopping power of this material different from that of a simple metal as Al. Though the average band gap of Si is small compared to the bandwidth of both valence and conduction bands, there are fewer low-energy excitation levels available than in the case of a FEG, and a lower energy loss is expected. Our calculations show that at low velocities the stopping power of $\mathrm{Si}$ is smaller than that of a FEG with $r_{s}=2.01$ by about $10 \%$. Also, the stopping power of $\mathrm{Si}$ is found not to be, at very small projectile velocities, proportional to the velocity, in agreement with experimental low-velocity stopping powers of this material [56].

As in the case of $\mathrm{Al}$, contributions from plasmon excitation to the stopping power of Si are found to be insensitive to the band structure, while contributions from electron-hole excitation yield a stopping power of this material which is just over the plasmon threshold about $20 \%$ lower than that of a FEG. At high velocities, well above the stopping maximum, all calculations are found to converge.

\section{B. Position-dependent stopping power}

We have carried out, from Eq. (34), calculations of the position-dependent electronic energy loss of protons in $\mathrm{Al}$ and $\mathrm{Si}$. In the case of $\mathrm{Al}$, calculations have been performed for slow ions moving along the (100) and (111) directions 24, showing that the existence of small electrondensity variations in this material result, through the presence of non-negligible off-diagonal elements in the interacting density-response matrix, in position-dependent corrections to the random stopping power of up to $10 \%$ and $20 \%$, respectively. The maxima in the stopping power for trajectories along the interstitial regions and the minima near the cores are associated with corresponding maxima and minima in the integrated electronic densities along the projectile trajectories. Nevertheless, a local-density approximation (LDA), according to which the position-dependent stopping power is obtained as that of a homogeneous electron gas with an electron density equal to the average electron density along the projectile trajectory, is found to predict corrections to the random stopping power which are, for low projectile velocities, too small.

A contour density plot of the square lattice containing both the projectile trajectory along the (110) direction in $\mathrm{Si}$ and the impact vector $\mathbf{b}$ is displayed in Fig. 12. The integrated density in the (110) channel varies from $r_{s}=1.49$ at the atomic row (full diagonal of the figure) to $r_{s}=3.37$ at the center of the channel (solid line with an arrow), thereby showing valence-electron density variations of up to $80 \%$ typical of covalent crystals like silicon.

In Fig. 13 we show, as a function of the projectile velocity, our full RPA calculation of the stopping power of Si for best channeled ions moving along the (110) direction. The stopping power of valence electrons in $\mathrm{Si}$ for these channeled ions is found to be, at intermediate velocities, about $20 \%$ smaller than the random stopping power. On the other hand, we note that the stopping maximum for channeled ions is located at the same velocity as in the case of random ions, while in the case of a homogeneous electron gas with an electron density equal to the average electron density along the channel would be located at a lower value of the velocity, as shown by the LDA calculation represented by a dotted line. We also note that within the LDA position-dependent corrections to the random stopping power for slow ions are predicted to be too small; for velocities above the stopping maximum, the local-density approximation yields unrealistic values for the energy loss of best channeled ions, which are far below our full band-structure calculations. At high velocities, position-dependent and random stopping powers are found to converge. 


\section{CONCLUSIONS}

We have presented a survey of current investigations of the impact of band structure effects on plasmon energies, plasmon linewidths, dynamic structure factors, and both random and position-dependent stopping powers of $\mathrm{Al}$ and Si.

New ab initio calculations for the plasmon lifetime in $\mathrm{Al}$ and $\mathrm{Si}$ have been presented. In the case of $\mathrm{Al}$, we find the full width at half maximum to be in the limit as $q \rightarrow 0$ of $0.2 \mathrm{eV}$, below the early calculation carried out by Sturm with use of the nearly free electron approximation [8]. The plasmon linewidth is found to increase as $q^{2}$ for small values of the momentum transfer $\mathbf{q}$, while it increases very quickly near the plasmon cutoff, in agreement with the experiment. A similar behaviour is found in the case of $\mathrm{Si}$, though plasmon linewidths in this material are found to be about five times wider than in $\mathrm{Al}$.

As for the random stopping power, we find that it is, at low velocities, smaller in Si than in Al, though they both have nearly the same valence electron density. At velocities just over the plasmon threshold they are both below the stopping power of a FEG with $r_{s} \sim 2$. At high velocities there are no band structure effects. The random stopping power of $\mathrm{Si}$ is found to be, at low and intermediate velocities, about 10\% smaller than that of $\mathrm{Al}$, in agreement with experimental measurements for either protons 57 or antiprotons 58.

Differences between random and position-dependent stopping powers of $\mathrm{Al}$ are found to be up to $10 \%$ for projectiles incident in the (100) direction and up to $20 \%$ for projectiles moving in the (111) direction. As for $\mathrm{Si}$, the stopping power for best channeled ions along the (110) direction is found to be diminished with respect to the random stopping power by about $20 \%$ at velocities near the stopping maximum.

[1] P. M. Echenique, F. Flores, and R. H. Ritchie, Solid State Phys. 43 (1990) 229.

[2] M. A. Kumakhov and F. F. Komarov, Energy loss and ion ranges in solids (Gordon and Breach Science Publishers, New York, 1981).

[3] D. Pines, Elementary excitations in solids (AddisonWesley, New York, 1963).

[4] H. Raether, in Excitations of Plasmons and Interband Transitions by Electrons, Vol. 88 of Springer Tracks in Modern Physics, Edited by G. Höhler (Springer, New York, 1980).

[5] D. F. DuBois and M. G. Kivelson, Phys. Rev. 186 (1969) 409.

[6] M. Hasegawa and M. Watabe, J. Phys. Soc. Japan 27 (1969) 1393.

[7] N. D. Mermin, Phys. Rev. B 1 (1970) 2362.
[8] K. Sturm, Z. Phys. B 28 (1977) 1.

[9] K. Sturm and L. E. Oliveira, Phys. Rev. B 24 (1981) 3054 .

[10] K. Sturm, Adv. in Phys. 31 (1982) 1.

[11] W. Ku and A. G. Eguiluz, Phys. Rev. Lett. 82 (1999) 2350.

[12] A. vom Felde, J. Sprösser-Prou, and J. Fink, Phys. Rev. B 40 (1989) 10181.

[13] R. H. Ritchie, Phys. Rev. 114 (1959) 644.

[14] F. F. Komarov and M. A. Kumakhov, Rad. Effects 22 (1974) 1.

[15] A. Desalvo and R. Rosa, J. Phys. C 10 (1977) 1595.

[16] H. Esbensen and J. A. Golovchenko, Nucl. Phys. A 298 (1978) 382.

[17] A. F. Burenkov, F. F. Komarov and M. A. Kumakhov, Phys. Status Solidi B 99 (1980) 417.

[18] O. H. Crawford and C. W. Nestor, Phys. Rev. A 28 (1983) 1260.

[19] A. Belkacem and P. Sigmund, Nucl. Instrum. Methods B 48 (1990) 29.

[20] P. L. Grande and G. Schiwietz, Phys. Lett. A 163 (1992) 439.

[21] J. J. Dorado and F. Flores, Phys. Rev. A 47 (1993) 3062.

[22] T. M. H. E. Tielens, Gerrit E. W. Bauer and T. H. Stoof, Phys. Rev. B 49 (1994) 5741.

[23] I. Campillo, J. M. Pitarke, A. G. Eguiluz and A. García, Nucl. Instrum. and Methods B 135 (1998) 103.

[24] I. Campillo, J. M. Pitarke, and A. G. Eguiluz, Phys. Rev. B 58 (1998) 10307.

[25] I. Campillo, J. M. Pitarke, and A. G. Eguiluz, to be published.

[26] M. Petersilka, U. J. Gossmann, and E. K. U. Gross, Phys. Rev. Lett. 76 (1996) 1212.

[27] E. K. U. Gross, F. J. Dobson, and M. Petersilka, Density Functional Theory (Springer, New York, 1996).

[28] C. C. Sung and R. H. Ritchie, Phys. Rev. A 28 (1983) 674; H. Esbensen and P. Sigmund, Ann. Phys. (NY) 201 (1990) 152; J. M. Pitarke, R. H. Ritchie, P. M. Echenique, and E. Zaremba, Europhys. Lett. 24 (1993) 613; J. M. Pitarke, R. H. Ritchie, and P. M. Echenique, Phys. Rev. B 52 (1995) 13883.

[29] P. Hohenberg and W. Kohn, Phys. Rev. 136 (1964) B864; W. Kohn and L. Sham, Phys. Rev. 140 (1965) A1133.

[30] J. Lindhard, K. Dan. Vidensk. Selsk. Mat.- Fys. Medd. 28, No. 8 (1954).

[31] K. S. Singwi, M. P. Tosi, R. H. Land, and A. Sjolander, Phys. Rev. 176 (1968) 589; K. S. Singwi, A. Sjolander, M. P. Tosi, and R. H. Land, ibid., 1 (1970) 1044; K. S. Singwi and M. P. Tosi, Solid State Phys. 36 (1981) 177.

[32] K. Utsumi and S. Ichimaru, Phys. Rev. B 22 (1980) 5203; S. Ichimaru, Rev. Mod. Phys. 54 (1982) 1017.

[33] A. Gold and L. Camels, Phys. Rev. B 48 (1993) 11622.

[34] D. M. Ceperley and B. J. Alder, Phys. Rev. Lett. 45 (1980) 566; J. Perdew and A. Zunger, Phys. Rev. B 23 (1981) 5048.

[35] H. J. Monkhorst and J. D. Pack, Phys. Rev. B 13 (1976) 5188.

[36] D. R. Hamann, M. Schluter, and C. Chiang, Phys. Rev. Lett. 43 (1979) 1494; D. R. Hamann, Phys. Rev. B 40 (1989) 2980. 
[37] P. M. Platzman, E. D. Isaacs, H. Williams, P. Zschack, G. E. Ice, Phys. Rev. B 46 (1992) 12943.

[38] E. N. Economou, Green's Functions in Quantum Physics (Springer-Verlag, Berlin. 1990).

[39] S. L. Adler, Phys. Rev. 126 (1962) 413.

[40] N. Wiser, Phys. Rev. 129 (1963) 62.

[41] G. A. Baker Jr., Essentials of Padé Approximants, (Academic Press, New York, 1975).

[42] K.-H. Lee and K. J. Chang, Phys. Rev. B 54 (1996) 8285. Padé approximants have also been used in Ref. [11], to compute the plasmon lifetime in potassium.

[43] A. A. Quong and A. G. Eguiluz, Phys. Rev. Lett. 70 (1993) 3955.

[44] K.-H. Lee and K. J. Chang, Phys. Rev. B 49 (1994) 2362.

[45] J. Sprösser, A. vom Felde, and J. Fink, Phys. Rev. B 40 (1989) 5799.

[46] P. C. Gibbons, S. E. Schnatterly, J. J. Risko, and J. R. Fields, Phys. Rev. B 13 (1976) 2451.

[47] J. Stiebling and H. Raether, Phys. Rev. Lett. 40 (1978) 1293.

[48] J. C. Phillips, Rev. Mod. Phys. 42 (1970) 317.

[49] R. Daling, W. Van Haeringen, and B. Farid, Phys. Rev. B 44 (1991) 2952; 45 (1992) 8970.

[50] A. J. Forsyth, T. W. Josefsson, and A. E. Smith Phys. Rev. B 54 (1996) 14355.

[51] S. G. Louie, J. R. Chelikowsky, and M. L. Cohen, Phys. Rev. Lett. 34 (1975) 155.

[52] A. Fleszar, A. A. Quong, and A. G. Eguiluz, Phys. Rev. Lett. 74 (1995) 590.

[53] Both the stopping power of valence electrons in the real solid and that of a FEG have been calculated with the integrations over the momentum transfer being extended from zero to $2.9 q_{f}$ and $2.1 q_{F}$ for $\mathrm{Al}$ and $\mathrm{Si}$, respectively. Since non-zero contributions to the stopping power come, within RPA, from all wave vectors that are smaller than $2\left(q_{F}+v\right)$, our calculated results are expected to underestimate the total stopping power of both jellium and band electrons for nearly all velocities under study. Nevertheless, we have found that differences, at large wave vectors, between full and FEG calculations are small, and our results provide, therefore, an accurate measure of the effect of the band structure on the random stopping power of valence electrons.

[54] J. C. Ashley, C. J. Tung, and R. H. Ritchie, Surf. Sci. 81 (1979) 409.

[55] M. C. Walske, Phys. Rev. 101 (1956) 940.

[56] J. F. Ziegler, J. P. Biersack, and U. Littmark, The stopping and range of ions in solids, Vol. 1 (Pergamon, New York, 1985).

[57] P. Bauer, Nucl. Instrum. Methods B 45 (1990) 673.

[58] S. P. Moller, E. Uggerhoj, H. Bluhme, H. Knudsen, U. Mikkelsen, K. Paludan, and E. Morenzoni, Phys. Rev. A 56 (1997) 2930.
FIG. 1. The RPA energy-loss function for $\mathrm{Al}$, for several wave vectors along the $(100)$ direction: $\mathbf{q}=(0.2,0,0) 2 \pi / a$

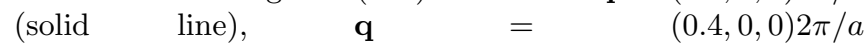
(long-dashed line), $\mathbf{q}=(0.6,0,0) 2 \pi / a$ (short-dashed line), and $\mathbf{q}=(0.8,0,0) 2 \pi / a$ (dashed-dotted line). The vertical dotted lines represent the corresponding results for a FEG with $r_{s}=2.07$, which are simply $\delta$ functions.

FIG. 2. RPA (solid line) and TDLDA (dotted line) plasmon excitation energies for wave vectors along the (100) direction in $\mathrm{Al}$. The corresponding calculations for a FEG are represented by dashed and dashed-dotted lines, as obtained within RPA and TDLDA, respectively. The experimental results are represented by triangles

FIG. 3. RPA plasmon linewidths of $\mathrm{Al}$ for wave vectors along the (100) direction. Solid circles and open triangles represent calculated linewidths without and with inclusion of plasmon decay through excitation of two electron-hole pairs. Solid triangles represent the experimental results.

FIG. 4. Scaled RPA (open triangles) and TDLDA (open circles) plasmon linewidths of $\mathrm{Al}$ for wave vectors along the (100) direction, as obtained with inclusion of plasmon decay through excitation of two electron-hole pairs. Solid triangles represent the experimental results.

FIG. 5. The RPA energy-loss function for Si, for several wave vectors along the (111) direction: $\mathbf{q}=(0.2,0.2,0.2) 2 \pi / a$ (solid line), $\mathbf{q}=(0.4,0.4,0.4) 2 \pi / a$ (dashed line), and $\mathbf{q}=(0.6,0.6,0.6) 2 \pi / a$ (dashed-dotted line). The vertical lines represent the corresponding results for a FEG with $r_{s}=2.01$, which are simply $\delta$ functions.

FIG. 6. RPA (solid line) and TDLDA (dotted line) plasmon excitation energies for wave vectors along the (111) direction in $\mathrm{Si}$. The corresponding RPA calculations for a FEG are represented by dashed lines. The experimental results are represented by triangles.

FIG. 7. RPA (solid circles) and TDLDA (open triangles) plasmon linewidths of $\mathrm{Si}$ for wave vectors along the (111) direction. Crystalline local-field effects have not been included in this calculation.

FIG. 8. The RPA (solid lines) and TDLDA (dashed lines) energy-loss function for $\mathrm{Al}$, for a large wave vector of magnitude $|\mathbf{q}+\mathbf{G}|=1.65 q_{F}$ along the direction (1,5/6,5/6). Plain solid and dashed lines represent RPA and TDLDA energy-loss functions of a FEG with $r_{s}=2.07$. 
FIG. 9. The RPA (solid lines) and TDLDA (dashed lines) energy-loss function for $\mathrm{Si}$, for a large wave vector of magnitude $|\mathbf{q}+\mathbf{G}|=1.95 q_{F}$ along the direction $(1,1,1)$. Plain solid and dashed lines represent RPA and TDLDA energy-loss functions of a FEG with $r_{s}=2.01$.

FIG. 10. Full band-structure calculation of the random stopping power of valence electrons in $\mathrm{Al}$ for protons $\left(Z_{1}=1\right)$, as a function of the projectile velocity. Solid and open circles represent the results obtained in the RPA and the TDLDA, respectively. RPA and TDLDA stopping powers of electrons in a FEG with $r_{s}=2.07$ are represented by solid and dashed lines, respectively.

FIG. 11. Full band-structure calculation of the random stopping power of valence electrons in $\mathrm{Si}$ for protons $\left(Z_{1}=1\right)$, as a function of the projectile velocity. Solid and open circles represent the results obtained in the RPA and the TDLDA, respectively. RPA and TDLDA stopping powers of electrons in a FEG with $r_{s}=2.01$ are represented by solid and dashed lines, respectively.

FIG. 12. Contour density plot of the valence electron density in the plane defined by the (100) and (010) vectors in Si.

FIG. 13. Full band-structure calculation of the RPA stopping power of valence electrons in Si for best channeled protons $\left(Z_{1}=1\right)$ in the (110) (solid circles), as a function of the projectile velocity. Solid and dashed lines represent the random stopping power of real Si and of a FEG with $r_{s}=2.01$, respectively. The dotted line represents the stopping power of a FEG with $r_{s}=3.37$. 
Figure 1

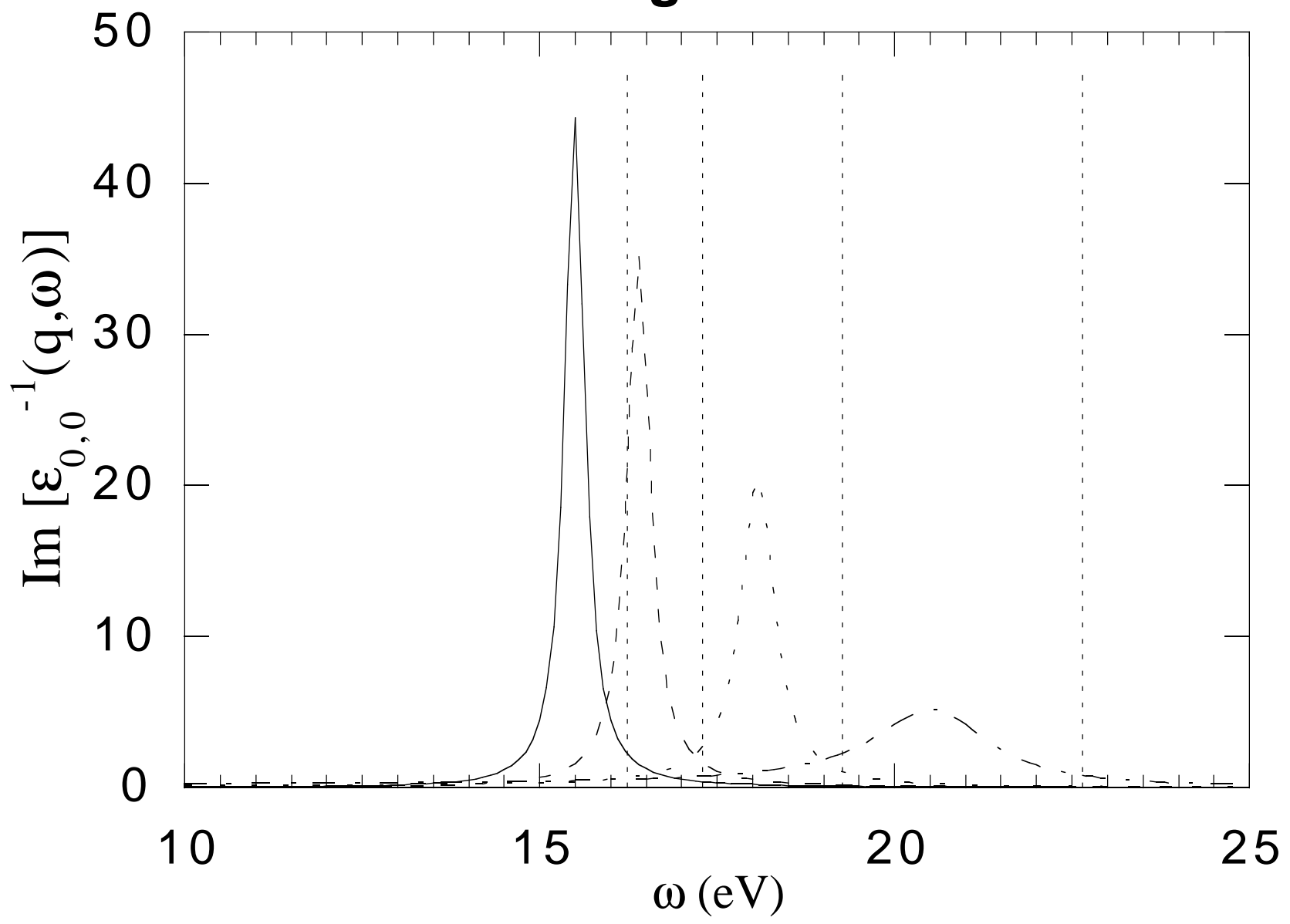


Figure 2

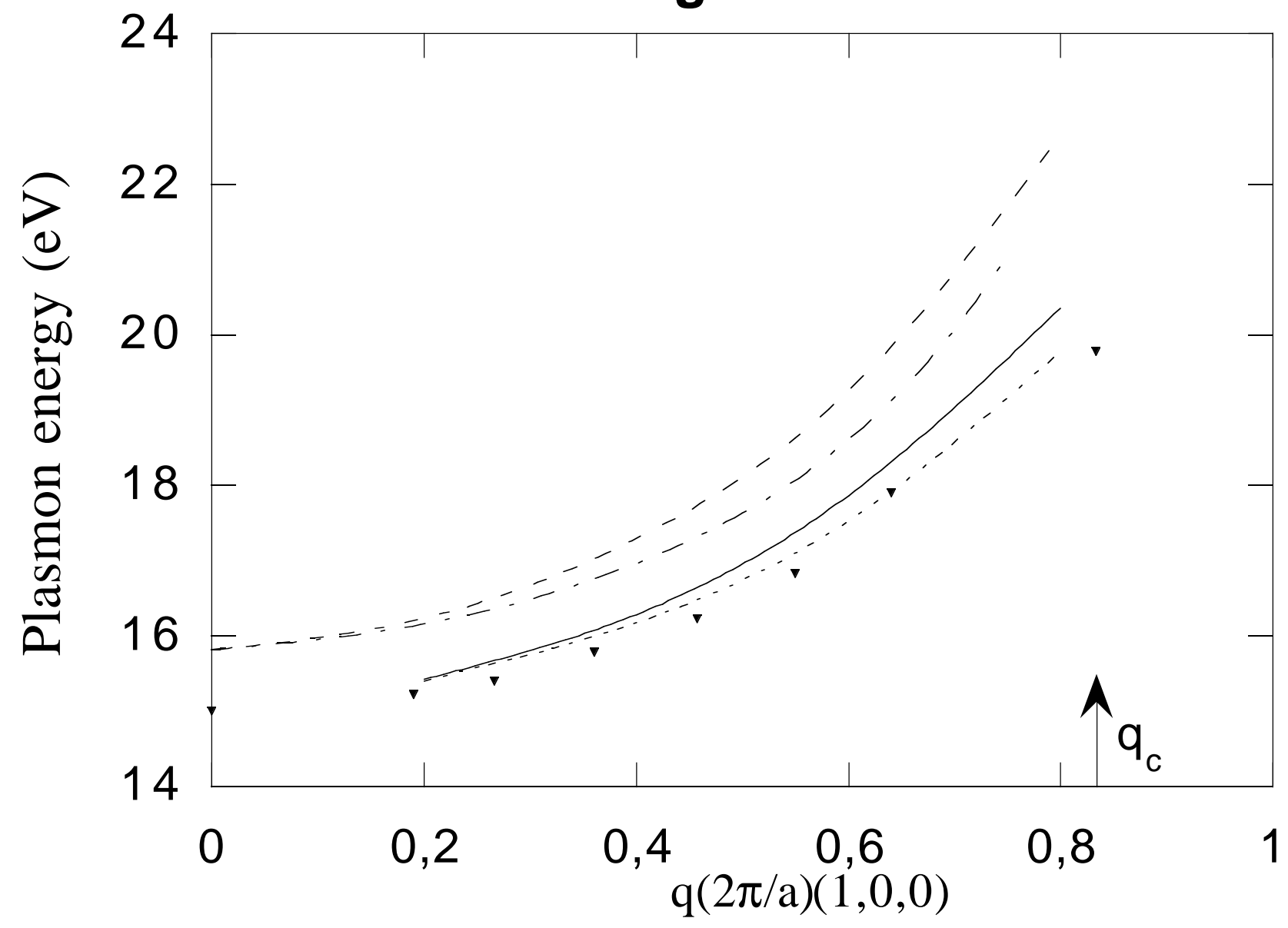


Figure 3

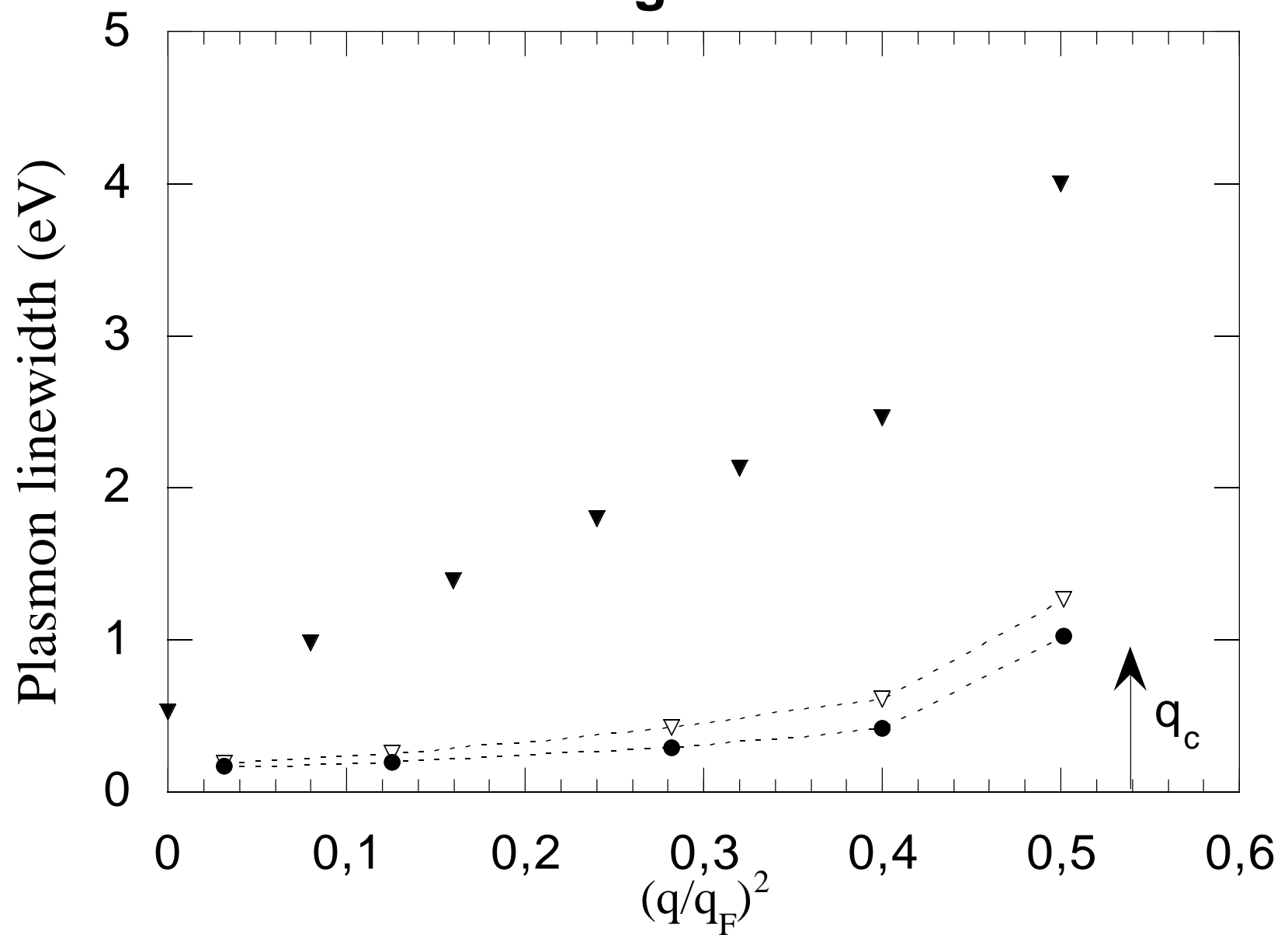


Figure 4

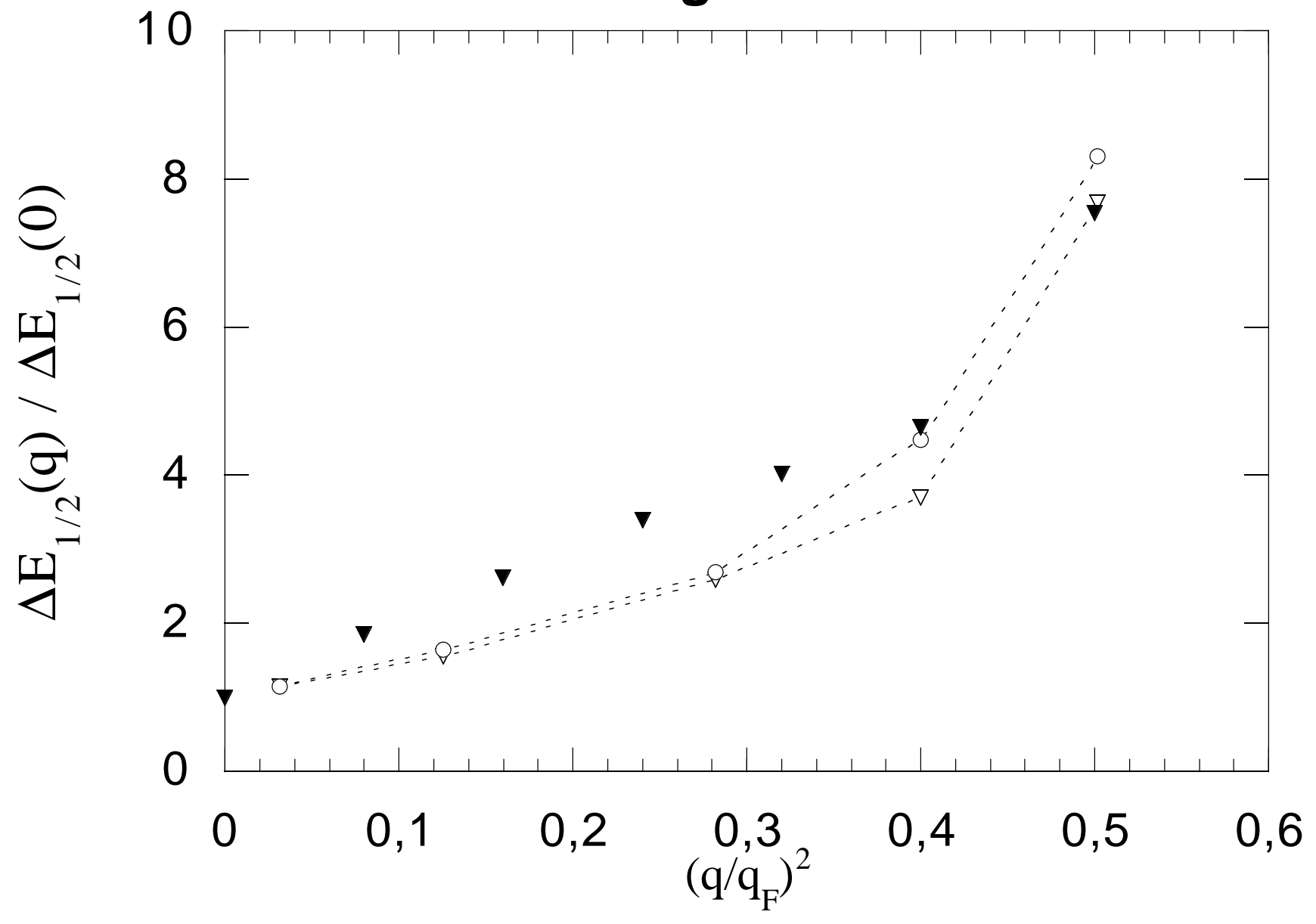


Figure 5

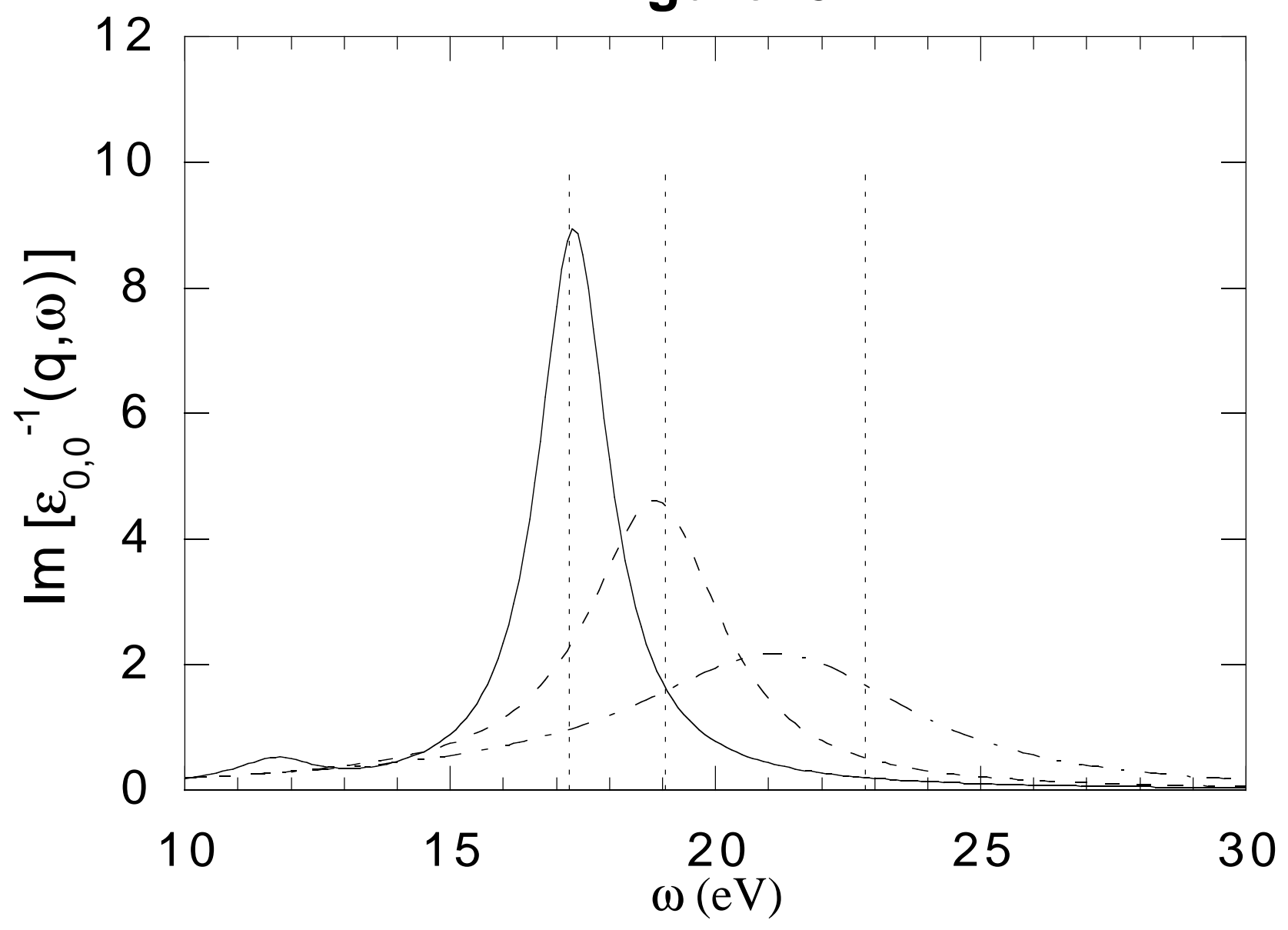


Figure 6

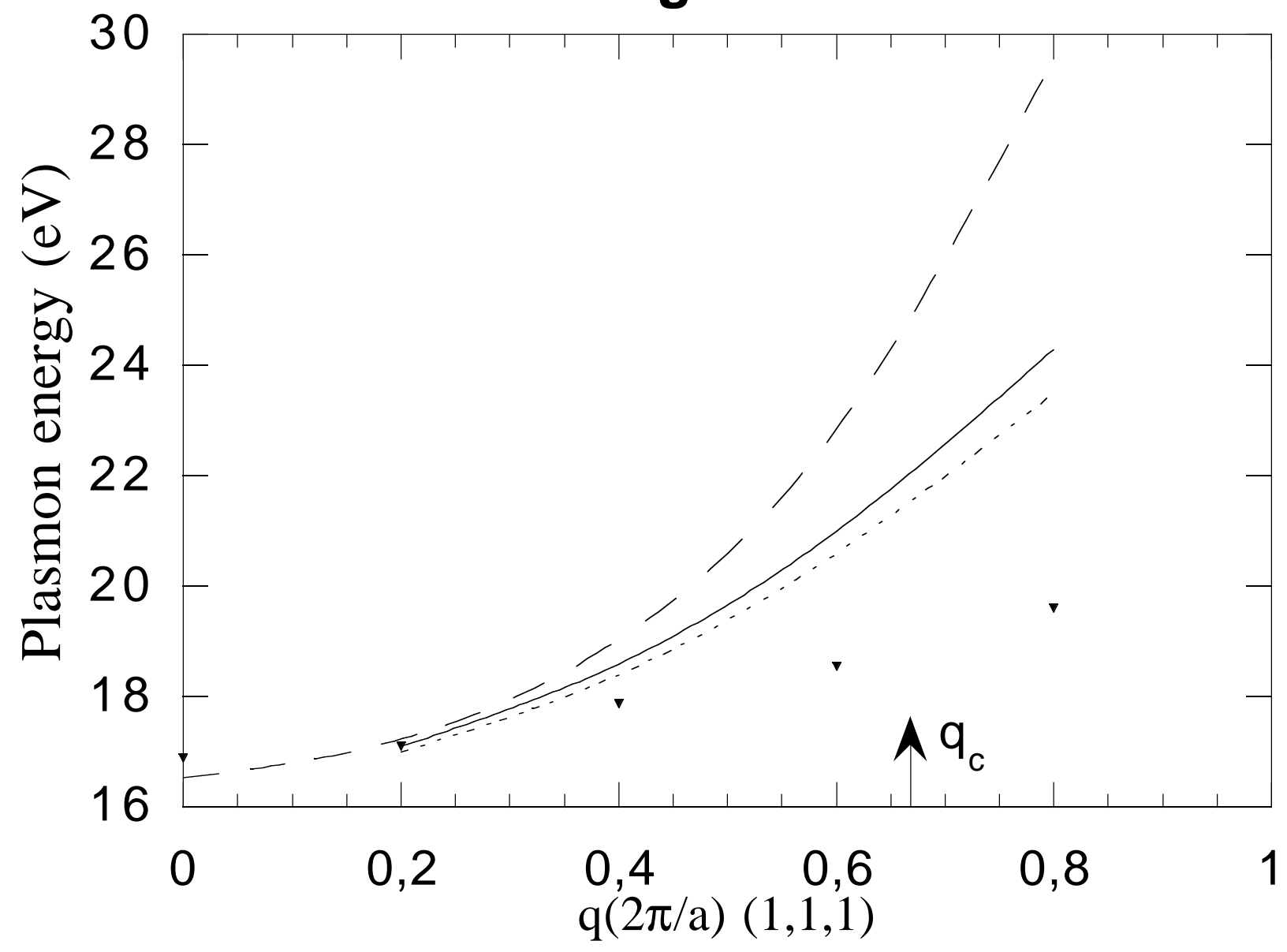


Figure 7

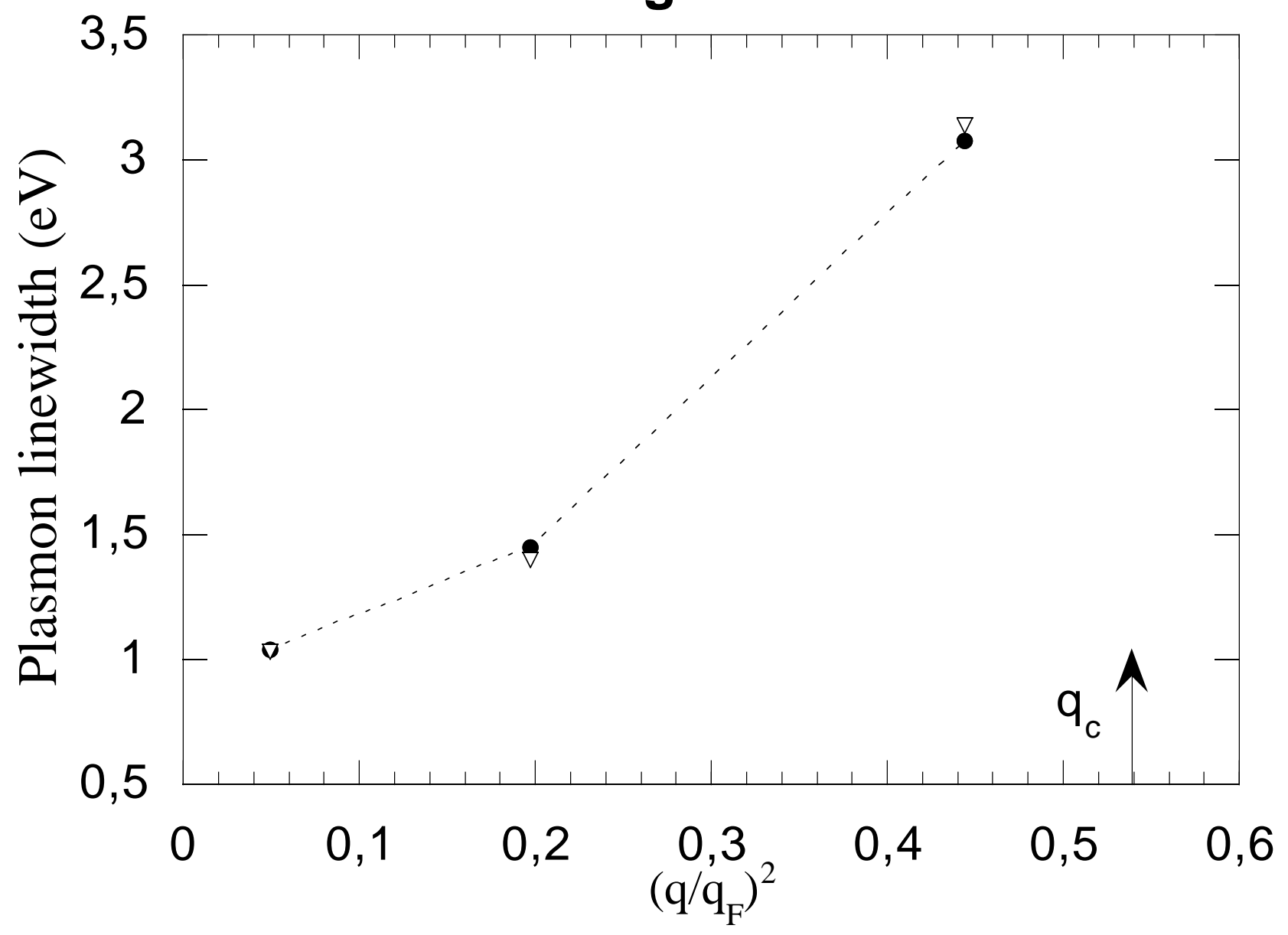


Figure 8

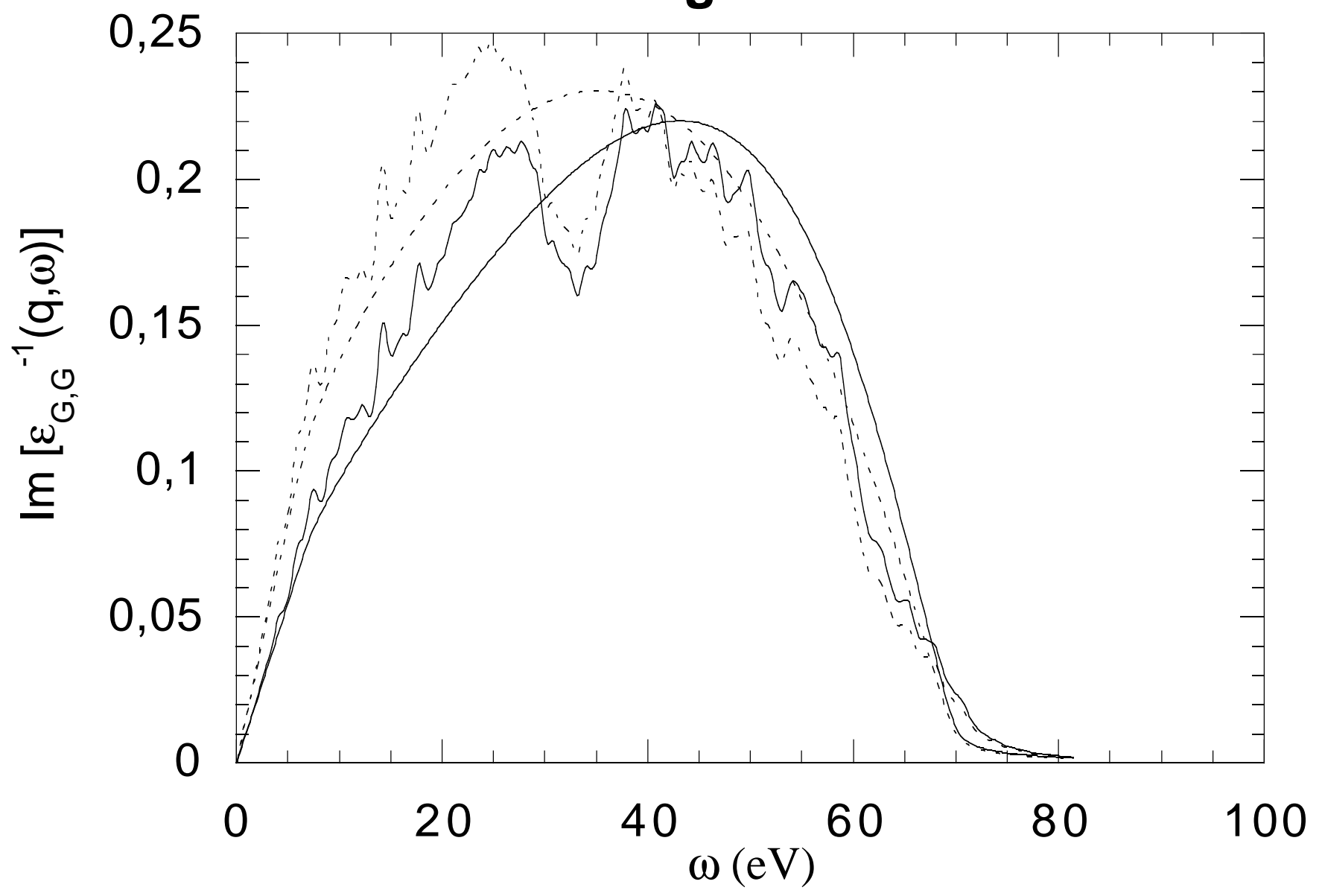


Figure 9

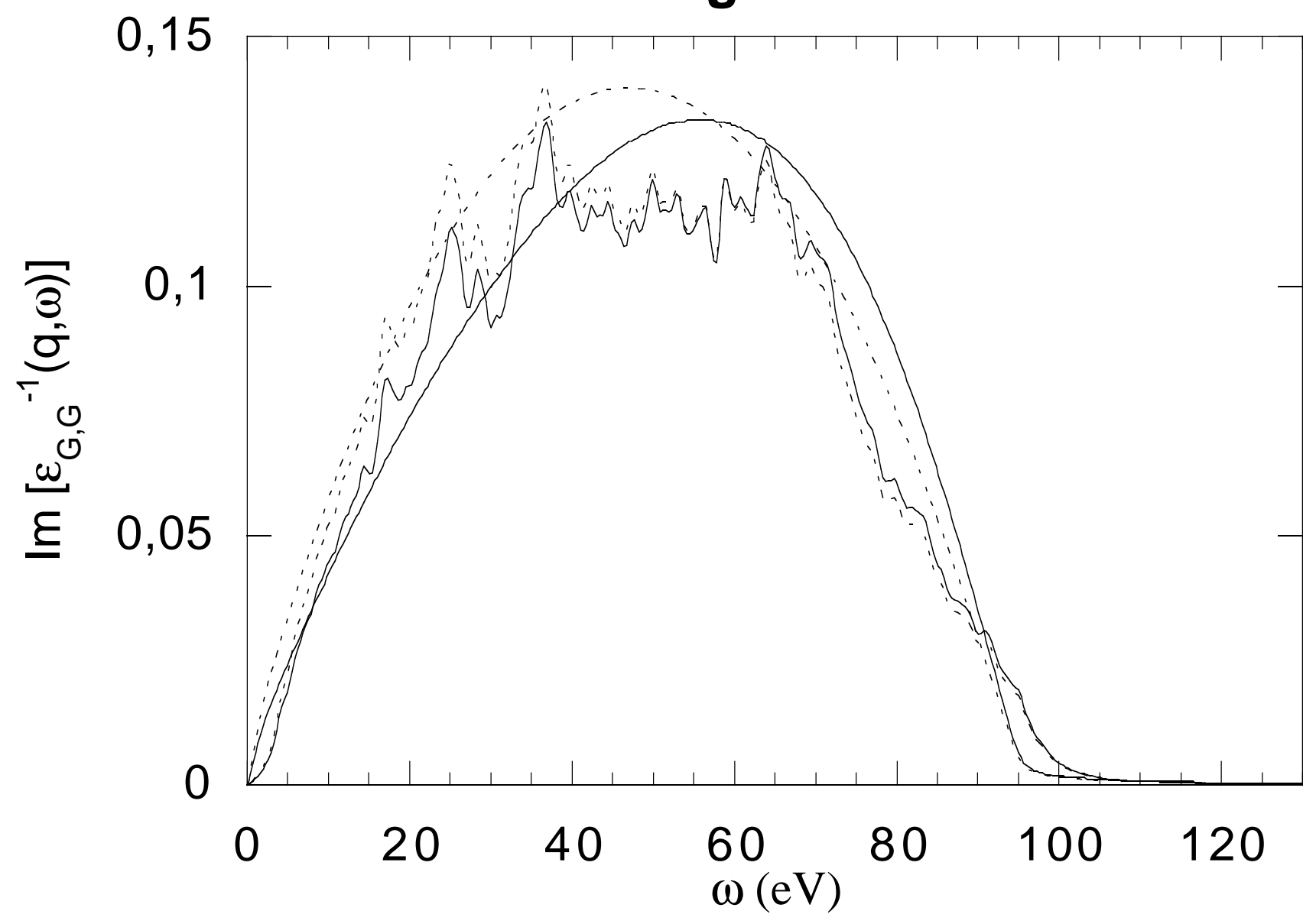


Figure 10

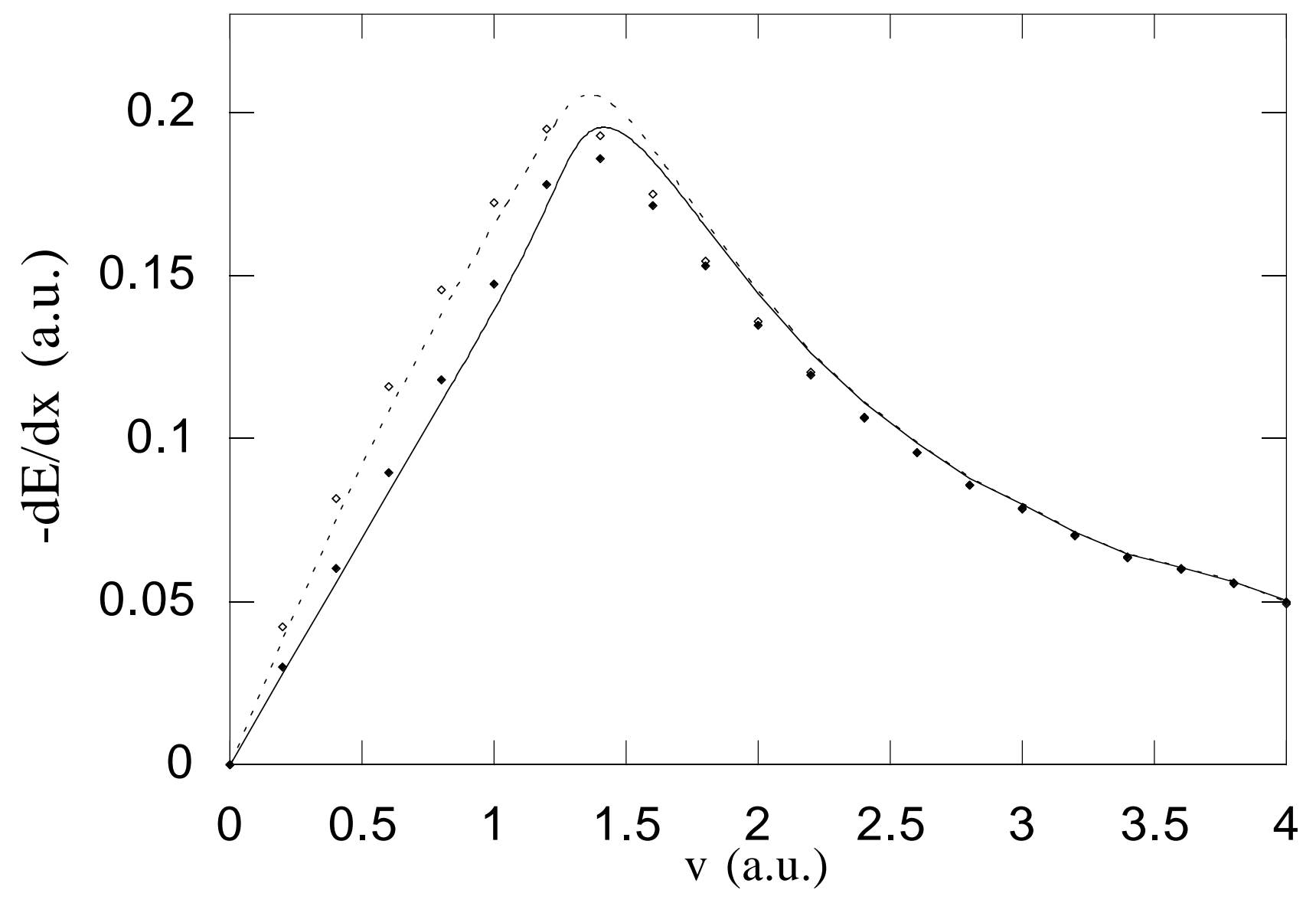


Figure 11

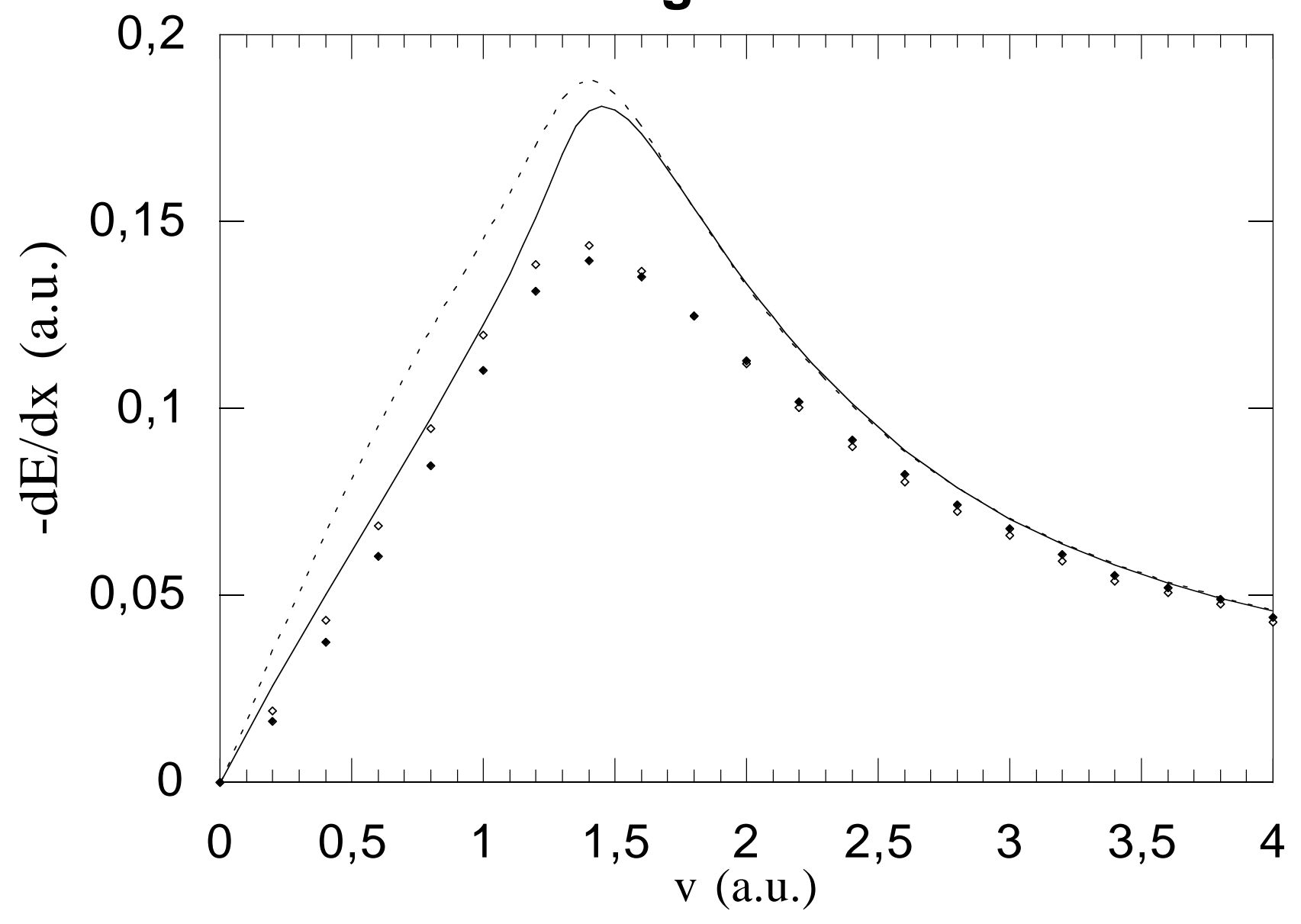


Figure 12

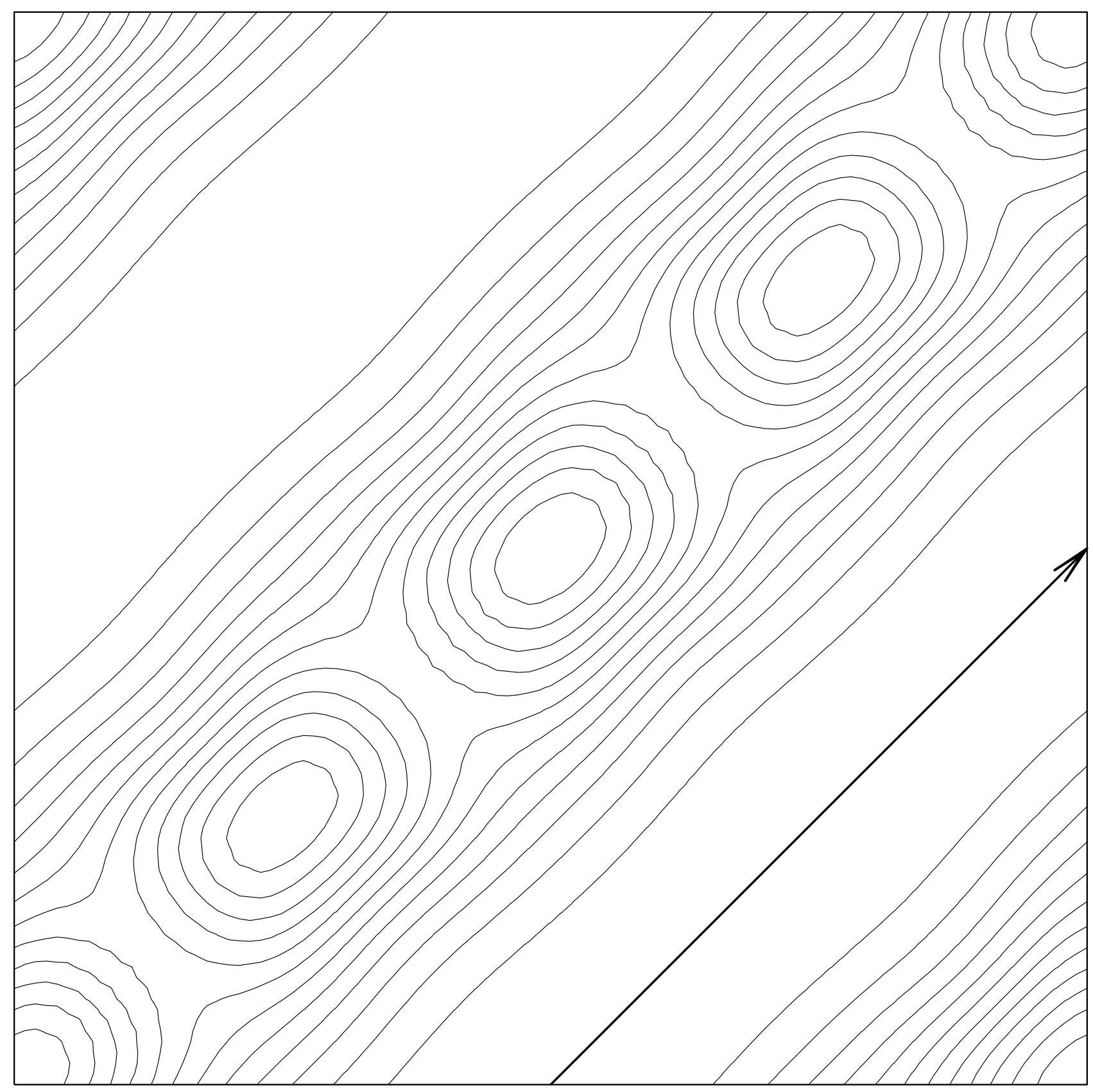


Figure 13

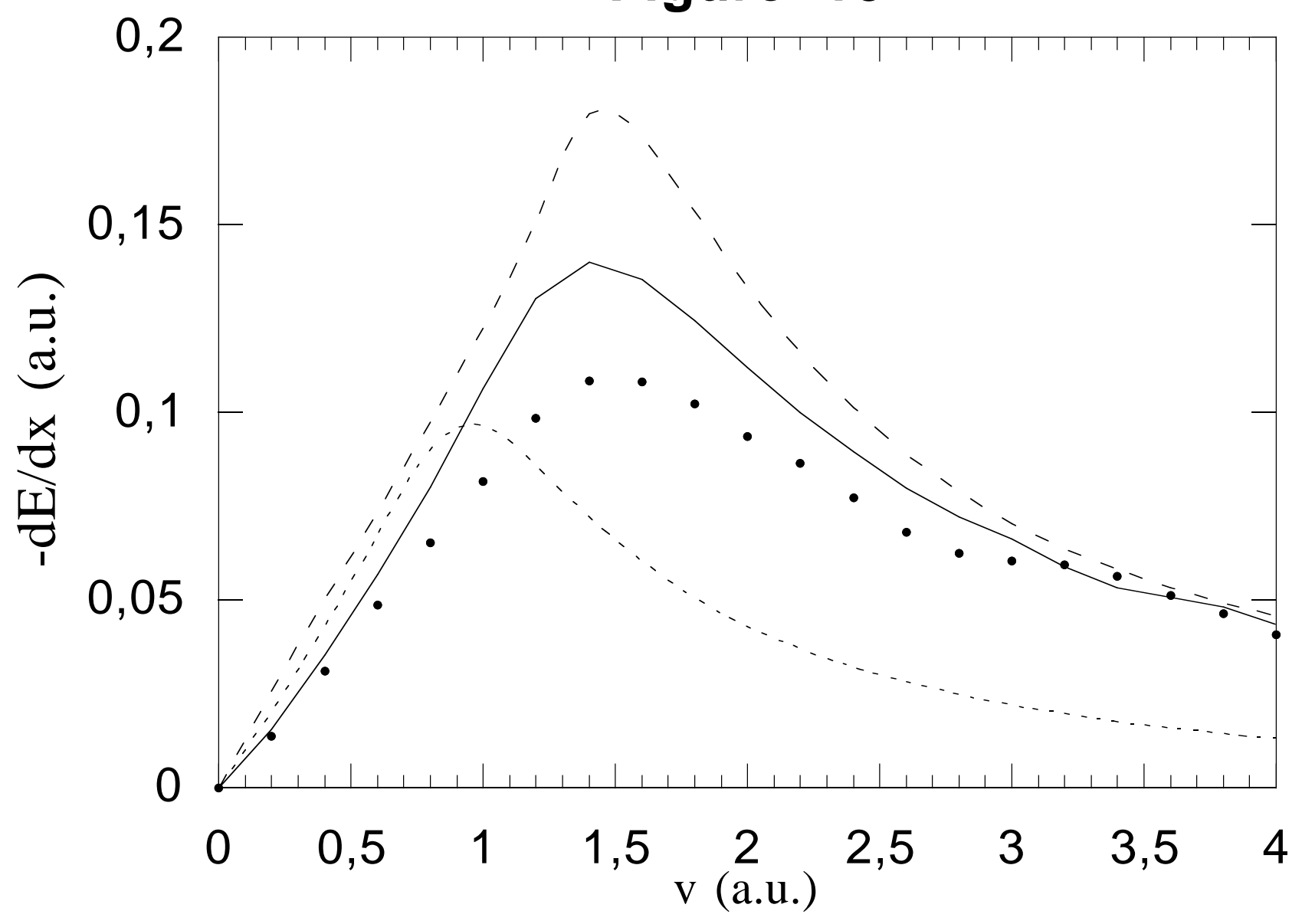

